\title{
Printed recyclable and self-poled polymer piezoelectric generators through single-walled carbon nanotube templating
}

Received 20th September 2019 Accepted 12th December 2019

DOI: $10.1039 /$ c9ee03059j

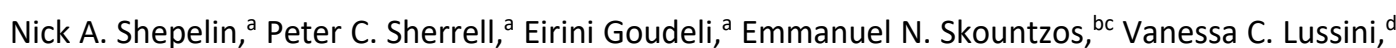
Greg W. Dicinoski, ${ }^{d}$ Joseph G. Shapter ${ }^{\mathrm{e}}$ and Amanda V. Ellis *a

\begin{abstract}
With an increasing global energy demand, along with a rising uptake of portable electronic devices, it is of great importance to investigate the viability of alternative energy harvesting technologies. Flexible piezoelectric generators (PEGs) are able to convert mechanical energy to electricity, making them an ideal candidate to decrease reliance on conventional energy sources and to power flexible, portable and implantable electronics. In this study, we show a low-energy production pathway for transparent PEGs based on poly(vinylidene fluoride-co-trifluoroethylene) (PVDF-TrFE) via shear-induced alignment of its dipoles through extrusion printing, complemented by spatial dipolar templating onto single-walled carbon nanotubes (SWCNTs) at low concentrations ( $<0.05 \mathrm{wt} \%$ ). The resulting composite PEGs show up to a $500 \%$ enhancement in the piezoelectric charge coefficient $d_{33}$ relative to extrusion printed pristine PVDF-TrFE, with similar enhancements in energy harvesting, exhibiting a power density of up to $20 \mu \mathrm{W} \mathrm{cm} \mathrm{cm}^{-3}$ at $0.02 \mathrm{wt} \%$ SWCNTs. The extrusion printed composite PEGs showed recyclability using only a green solvent (acetone) and were found to exhibit piezoelectric energy harvesting with a power density of up to $71 \mu \mathrm{W} \mathrm{cm} \mathrm{cm}^{-3}$ upon reprinting, overcoming two of the most significant hurdles towards commercial production of flexible PEGs.
\end{abstract}

\section{Introduction}

Wearable electronic devices are ubiquitous in modern society, providing personalised information and sensing to an estimated $22 \%$ of US adults in 2019 and growing year by year. ${ }^{1}$ Similar to trends over the last 20 years in smartphone technology, there is a desire for the next generation of wearable electronics to be lighter and smaller, with longer lifetimes. A major bottleneck toward achieving miniaturisation in electronic devices is the power supply, conventionally based on electrochemical devices, which require the incorporation of fluid electrolytes and manual recharging. ${ }^{2}$ Therefore, finding lightweight and low footprint alternatives for power delivery is crucial in the development of next generation wearable electronics. ${ }^{3}$ Many sustainable technologies have been proposed as alternative energy sources, including photovoltaic, thermo-, pyro-, and mechano-electric technologies. ${ }^{4-7}$ However, for devices with low power requirements, flexible piezoelectric generators (PEGs) have emerged as ideal candidates, requiring no manual recharge, being completely solid-state, and being relatively lightweight.

\footnotetext{
a. Department of Chemical Engineering, The University of Melbourne, Parkville, Victoria 3010, Australia. Email: amanda.ellis@unimelb.edu.au.

b. Department of Chemical Engineering, University of Patras, Greece.

c. FORTH/ICE-HT, Patras, GR 26504, Greece.

d. Note Issue Department, Reserve Bank of Australia, Craigieburn, Victoria 3064, Australia.

e. Australian Institute for Bioengineering and Nanotechnology, The University of Queensland, Brisbane, Queensland 4072, Australia.

+ Electronic Supplementary Information (ESI) available. See DOI: 10.1039/ c9ee03059j
}

While piezoelectricity as a means of harvesting energy has been found in literature as early as 1961, the desire for renewable and independent energy generators has provoked a great revival of interest within the academic community over the past several years. ${ }^{8}$ For the purposes of energy harvesting, ceramic and polymer materials have shown the highest promise, however ceramic devices are rigid, brittle, and often lack biocompatibility.7,9,10 Conversely, polymeric materials are flexible and biocompatible, with the potential for transparency, thus increasing their scope for reliable commercial use. ${ }^{9,11,12}$ In particular, fluoropolymers have shown the highest piezoelectric energy harvesting capability of all flexible polymer PEGs due to their high dipole polarisation, on the order of $131 \mathrm{mC} \mathrm{m}^{-2}$, between their hydrogen and fluorine atoms perpendicular to the carbon backbone. ${ }^{13}$ The piezoelectric figure of merit (FOM) for thin films, corresponding to the product of the piezoelectric charge $\left(d_{33}\right)$ and voltage $\left(g_{33}\right)$ coefficients, $d_{33} g_{33}$, has been experimentally validated to show similar values for both ceramic materials and polymers. ${ }^{14}$ Piezoelectric polymers, dominated largely by fluoropolymers, have shown lower values for the harvested $d_{33}$ and volumetric power density $\left(P_{D}\right)$; however, the reports of the $g_{33}$ have shown significantly higher values relative to the ceramic materials. ${ }^{7}$ Similar FOM values, coupled with their ease of use in flexible, wearable and implantable electronic devices, make fluoropolymers an attractive option for energy harvesting applications. For wearable electronics, flexible, transparent and light weight devices are paramount. Polymer-based PEGs are far superior in these requirements compared to their ceramic counterparts. 
However, one of the major challenges currently preventing widespread use of fluoropolymers in energy harvesting is the cost to process the polymers into the piezoelectric phase and subsequently align their inherent dipoles, which is also a requirement for efficient PEGs.7,9

Polycrystalline dielectric fluoropolymers such as poly(vinylidene fluoride) (PVDF) can be deposited into the nonpolar and non-piezoelectric a phase (trans-gauche-transgauche' polymorph, TGTG') when low energy techniques such as solvent-casting are employed. ${ }^{15}$ The conversion of the $\alpha$ phase to the polar phases, including the highly piezoelectric $\beta$ phase (TTTT polymorph) and the partially piezoelectric $\gamma$ phase (TTTGTTTG' polymorph), has become a widespread research area, whereby multiple authors have claimed to achieve $\beta$ phase fractions as high as $100 \% .7,16,17$ The utilisation of a comonomer during the polymerisation process, such as trifluoroethylene (TrFE), has been further reported to enhance the nucleation of fluoropolymers into the piezoelectric phases during deposition. ${ }^{15}$ At TrFE fractions between 25 mol\% and 30 mol\% relative to VDF, the copolymer films have been demonstrated to contain a higher $\beta$ phase fraction relative to equivalent PVDF films, arising from the steric interactions between the third fluoride atom in the TrFE. ${ }^{9}$ Nonetheless, an additional processing step called poling is required to spatially align the dipoles of the piezoelectric phases for high energy conversion efficiencies. ${ }^{9,18}$ In the poling process, generally undertaken under vacuum and at temperatures between the Curie temperature $\left(T_{C}\right)$ and melting temperature $\left(T_{m}\right)\left(90^{\circ} \mathrm{C}\right.$ and $110^{\circ} \mathrm{C}$, respectively), electric fields between $2 \mathrm{MV} \mathrm{m}^{-1}$ and 400 $\mathrm{MV} \mathrm{m^{-1 }}$ are applied across the material.7,9,19 Each part of this step utilises a significant amount of energy and is difficult to scale to roll-to-roll processes, currently only undertaken by Measurement Specialties, Inc. ${ }^{20}$

In order to improve piezoelectric outputs from fluoropolymers, researchers have incorporated various conducting, semiconducting, insulating and piezoelectric additives into fluoropolymers. ${ }^{7,15}$ Additionally, a range of additive morphologies have been trialled such as nanoparticles, ${ }^{21-25}$ nanowires/nanotubes ${ }^{26-36}$ and planar nanomaterials. ${ }^{37,38}$ The majority of the studies have reported enhancements in the $\beta$-phase fraction, crystallinity or both. ${ }^{22-}$ 25,28,32,37-39 One-dimensional (1D) carbon nanotube (CNT) additives are of increasing interest due to their decreased toxicity relative to their inorganic counterparts and provide mechanical reinforcement while remaining flexible at concentrations <1 wt\%, important for both wearable and implantable energy harvesters. ${ }^{40-42}$ CNTs have been shown to potentially aid $\beta$ phase nucleation. ${ }^{32,39,43}$ Fabrication methods including solvent casting (poling at $60 \mathrm{MV} \mathrm{m}^{-1}$ electric field), electrospinning ( $7 \mathrm{MV} \mathrm{m}^{-1}$ ) and spray coating have been used to produce CNT/PVDF composites, with the highest energy harvesting enhancements found at approximately $0.1 \mathrm{wt} \%$ CNTs. ${ }^{30,44}$ More recently, Lee et al. ${ }^{39}$ have investigated the energy harvesting characteristics of spray coated multi-walled CNT (MWCNT)/PVDF composites without poling. The unpoled MWCNT/PVDF PEGs produced a maximum open circuit voltage at $1.2 \mathrm{~V}$ and short circuit current at $3.8 \mathrm{nA}$, approximately $30 \%$ of the voltage output and $1 \%$ of the current output, respectively, of poled MWCNT/PVDF composites.

To fabricate electroactive fluoropolymers without poling, extrusion printing has been performed, albeit with significantly lower piezoelectric FOM. Bodkhe et al.45,46 have 3D printed a composite containing piezoelectric $\mathrm{BaTiO}_{3}$ nanoparticles and PVDF. More recently, our group has 3D printed poly(vinylidene fluoride-co-trifluoroethylene) (PVDF-TrFE) with no additives, showing partial shear-induced polarisation in PVDF-TrFE for unpoled PEGs. ${ }^{47}$ In addition to inducing polarisation of the fluoropolymers, the 3D printing technique allows for PEGs to be fabricated into complex shapes and structures. Recent advances in 3D printing have demonstrated printing on moving, rough and uneven surfaces, enabling conformal printing directly onto skin. ${ }^{48}$

This work presents significant advances in the design and manufacturing of flexible piezoelectric energy harvesters. Through the combination of Molecular Dynamics modelling of the dipole direction, solvent evaporation-assisted extrusion printing, and the selected use of SWCNTs as additives, we demonstrate efficient, low voltage switching, and high power density PVDF-TrFE piezoelectric energy harvesters that can be used as produced. The use of this intelligent design and bespoke processing enables a preferential dipole alignment which leads to the complete elimination of the electrical poling process, an expensive and arduous process that is unavoidable in the current manufacture of polymer-based piezoelectric generators. The printed devices show no significant change in the piezoelectric $\beta$-phase through SWCNT addition; however, exhibit dramatic increases in the $d_{33}$ and $P_{D}$ at the optimal SWCNT concentration, confirming the increased performance arises from our theoretically predicted dipole alignment. The printed piezoelectric generators are transparent and flexible with figures of merit comparable to commercially available poled PVDF. We also show that our piezoelectric PVDF-TrFE energy harvesters are recyclable in green solvents such as acetone, a never before observed phenomenon, and can be reprocessed into new piezoelectric materials without loss of properties. These key points provide a fundamental breakthrough in the design and fabrication of low cost and recyclable flexible PEGs, opening up a path to their broad application for low power flexible electronics.

\section{Results and discussion}

\section{Inks, printing and optical properties}

For extrusion printing, PVDF-TrFE inks were prepared by dissolving PVDF-TrFE (30 wt\%) in a solvent mixture containing acetone (60 vol\%) and $\mathrm{N}, \mathrm{N}$-dimethylformamide (DMF) (40 vol\%). For the SWCNT/PVDF-TrFE inks, the acetone:DMF (60:40 vol\%) solvent mixture contained suspended SWCNTs at concentrations ranging from $0.005 \mathrm{wt} \%$ to $0.500 \mathrm{wt} \%$ relative to the PVDF-TrFE (Fig. S1, ESIt).

The rheological properties of the PVDF-TrFE (0.000 wt\%) and SWCNT/PVDF-TrFE (0.005 wt\% to $0.200 \mathrm{wt} \%)$ inks were studied in order to determine their printability, shown in Fig. S2 (ESI ${ }^{+}$). 
For the SWCNT/PVDF-TrFE inks, the viscosity values showed low deviation from that of the PVDF-TrFE ink, while the viscosity profile retained non-Newtonian, shear thinning properties, which are optimal for extrusion printing. ${ }^{47}$ The results showed a zero shear viscosity $\left(\eta_{0}\right)$ between $260 \mathrm{~Pa} \cdot \mathrm{s}$ and $638 \mathrm{~Pa} \cdot \mathrm{s}$, while the viscosity approaching printing shear rates was between 2.9 $\mathrm{Pa} \cdot \mathrm{s}$ and $4.8 \mathrm{~Pa} \cdot \mathrm{s}$, corresponding to the values for the inks at rest before and after printing, and during the extrusion step, respectively.

Fig. 1a shows a composite photograph of the printed single layer films with increasing SWCNT concentration from left $(0.000 \mathrm{wt} \%)$ to right $(0.200 \mathrm{wt} \%)$, placed on top of a graphic to demonstrate their optical properties. The films containing SWCNT concentrations below 0.050 wt\% were indistinguishable from the PVDF-TrFE control film, while those at 0.050 wt\% and above exhibited an increasing dark colouration. The films appeared homogeneous and did not show visible aggregation at SWCNT concentrations up to $0.200 \mathrm{wt} \%$, suggesting that the SWCNTs were stable during the printing and drying processes. The visible wavelength transmittance and haze of the films, measured between $380 \mathrm{~nm}$ and $780 \mathrm{~nm}$, are shown as a function of SWCNT concentration in Fig. 1b. At low SWCNT concentrations $(<0.050 \mathrm{wt} \%)$, the composite films absorbed minimal light and were transparent over the entire measured spectrum (Fig. S3, ESI + ). The haze values, shown in Fig. 1b, were found to be constant at $3.5 \%$ below 0.050 wt\% SWCNTs, further showing the stability of dispersed SWCNTs in the PVDF-TrFE with minimal deviation from the spectrum of the pure polymer. However, at SWCNT concentrations $>0.050$ wt\%, increased haze was observed, attributed to SWCNT bundling (see Fig. S4, ESI†).

Fig. 1c shows the helium ion microscopy (HIM) images of the surface of the extrusion printed PVDF-TrFE films as a function of (a) Increasing SWCNT concentration (wt\%)
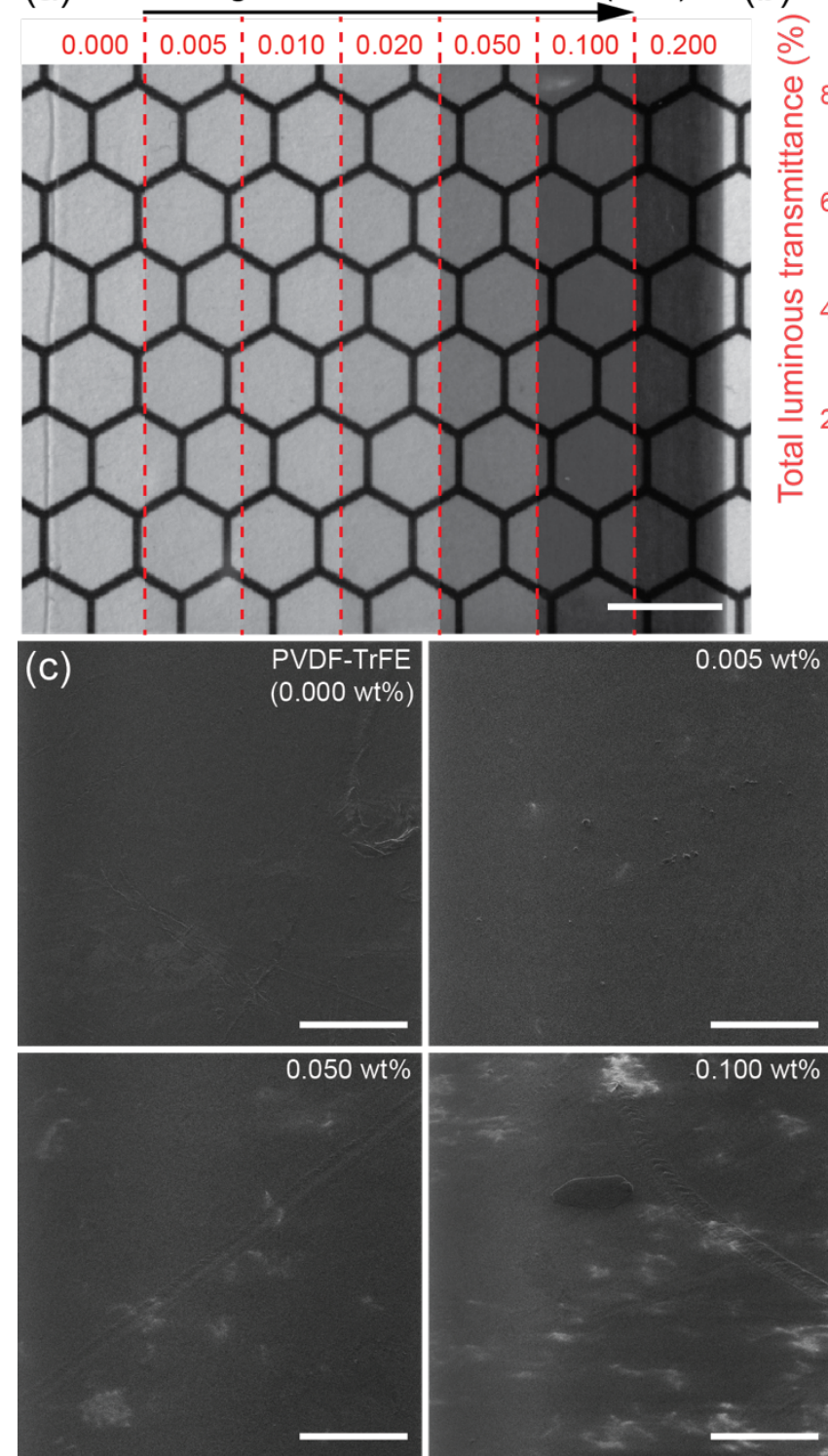

(b) 100
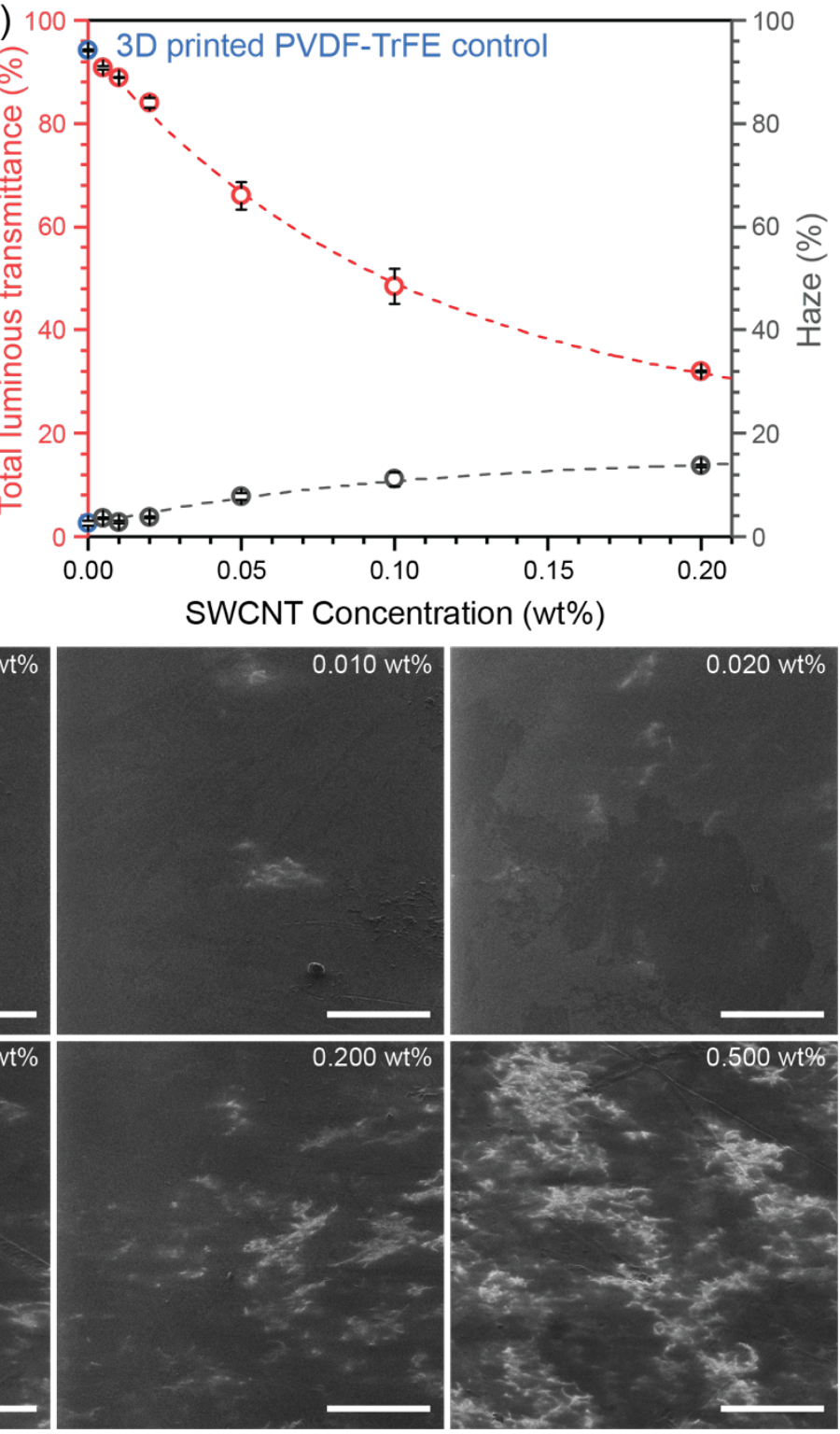

Fig. 1 (a) Optical images demonstrating the transparency of extrusion printed PVDF-TrFE films with increasing SWCNT concentration (0.000 wt\% to 0.200 wt\%) from left to right (scale bar represents $5 \mathrm{~mm}$ ). (b) Visible wavelength transmittance and haze as a function of SWCNT concentration (blue datapoints denote the PVDF-TrFE control). (c) Helium ion microscopy (HIM) images showing the surface of the films for PVDF-TrFE (0.000 wt\%) and SWCNT/PVDF-TrFE (0.005 wt $\%$ to 0.500 wt\%) (scale bar represents $10 \mu \mathrm{m})$. 

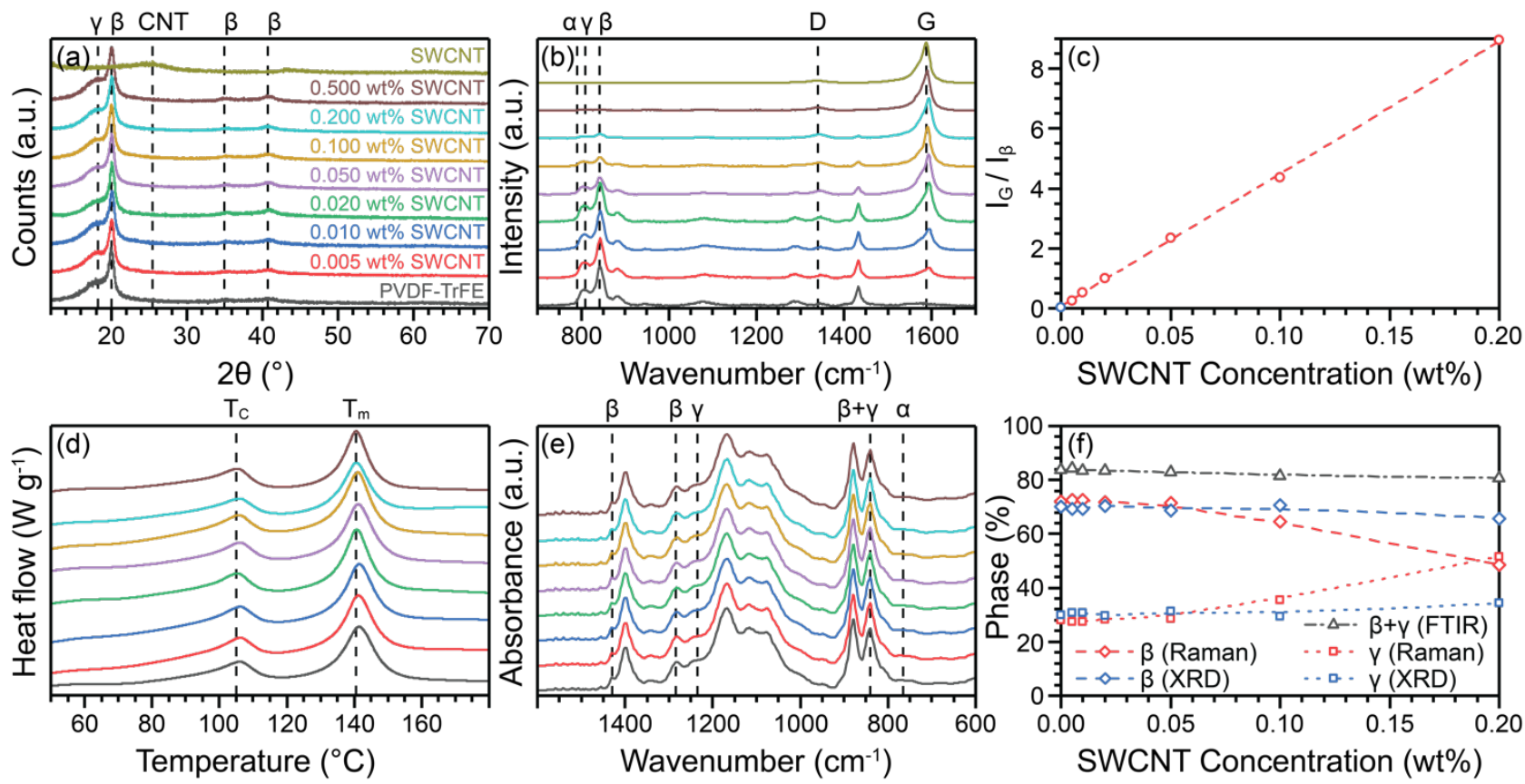

Fig. 2 Analysis of extrusion printed PVDF-TrFE films with SWCNT concentrations between 0.000 wt $\%$ and 0.500 wt\%, showing (a) X-ray powder diffraction (XRD) spectra. (b) Raman spectra. (c) Ratio of the intensities of the G-band peak relative to the $\beta$ phase peak from Raman spectra. (d) Differential scanning calorimetry (DSC) heating traces. (e) Attenuated total reflectance Fourier-transform infrared (ATR-FTIR) spectra. (f) The relative phase ratios calculated from ATR-FTIR spectra (grey), Raman spectra (red) and XRD spectra (blue) for the $\beta+\gamma$ phase (triangle), $\beta$ phase (diamond) and $\gamma$ phase (square).

SWCNT concentration. The overall surface morphology was found to be free of visible porosity, confirming the formation of solid, non-porous thin films upon printing. The HIM technique allows for strong contrast between the dielectric, nonconductive PVDF-TrFE surface, shown as dark regions in the micrographs, and the conducting SWCNTs shown in the light regions. This contrast decreased for SWCNTs embedded further below the surface. Minimal bundling and aggregation of the SWCNTS was observed on the surface of the samples with concentrations below 0.050 wt\%; however, larger bundles of nanotubes were visible at higher concentrations with the 0.500 wt\% film exhibiting continuous networks of SWCNTs over its entire surface.

\section{Phase determination and materials properties}

The PVDF-TrFE and SWCNT/PVDF-TrFE films were characterised to investigate the polymer phase behaviour with SWCNT loading relative to PVDF-TrFE. Fig. 2a shows the $\mathrm{X}$-ray diffraction (XRD) spectra for the printed films. The broad peak at $18.1^{\circ} 2 \theta$ corresponds to the $(110 / 200)$ paraelectric (PE) phase reflection and the peak at $20.2^{\circ} 2 \theta$ corresponds to the $(110 / 200)$ ferroelectric (FE) phase reflection commonly attributed to the $\beta$ phase of PVDF-TrFE. ${ }^{19}$ The two lower intensity peaks at $35.0^{\circ} 2 \theta$ and $40.8^{\circ} 2 \theta$ were attributed to the (001) FE reflection and (111/201), (400/220) FE reflections, respectively, further indicating the presence of the $\beta$ phase within the polymer matrix. ${ }^{49,50}$

The Raman spectra for the SWCNTs, PVDF-TrFE films and SWCNT/PVDF-TrFE (0.005 wt\% to $0.500 w t \%)$ films are shown in Fig. 2b. All polymer samples showed peaks characteristic of fluoropolymers at $1432 \mathrm{~cm}^{-1}$ and $880 \mathrm{~cm}^{-1}$, as well as the characteristic $\beta$ phase peak at $842 \mathrm{~cm}^{-1}$ and $\gamma$ phase peak at 810 $\mathrm{cm}^{-1} .7,51,52$ There was a notable absence of the non-polar $\alpha$ phase peak typically observed at $794 \mathrm{~cm}^{-1} .{ }^{53}$ The Raman spectra of the SWCNT/PVDF-TrFE films all showed a strong contribution from the characteristic SWCNT graphitic G-band $\left(1587 \mathrm{~cm}^{-1}\right)$, along with a slight contribution from the disordered defect Dband $\left(1340 \mathrm{~cm}^{-1}\right) .{ }^{54}$ The ratio of the intensity of the G-band $\left(I_{G}\right)$ to the intensity of the $\beta$ phase peak $\left(I_{B}\right)$ was plotted as a function of SWCNT concentration and is shown in Fig. 2c. The linear trend up to a 0.200 wt\% SWCNT concentration suggested a homogeneous dispersion.

Fig. $2 \mathrm{~d}$ shows the differential scanning calorimetry (DSC) traces for the SWCNT/PVDF-TrFE (0.000 wt\% to 0.500 wt\%) films during a heating cycle. The position of the $T_{m}$ was at approximately $140.5^{\circ} \mathrm{C}$, within the typical range for PVDF-TrFE (75 mol\% VDF:25 mol\% TrFE, $420 \mathrm{kDa}$, Solvay). The FE to PE transition, represented as the $T_{C}$, was found to be $105{ }^{\circ} \mathrm{C}$ suggesting piezoelectric stability for temperatures up to approximately $100{ }^{\circ} \mathrm{C}$.

The crystallinity $\left(\chi_{\mathrm{c}}\right)$ of the SWCNT/PVDF-TrFE $(0.000 \mathrm{wt} \%$ to 0.500 wt\%) films was calculated from the DSC traces using eqn (1) with values shown in Table 1.

$$
\chi_{C}(\%)=\frac{\Delta H_{m}}{\Delta H_{0}} \times 100
$$

Here, the enthalpy of melting $\left(\Delta H_{m}\right)$ was calculated from the mass-normalised area of the $T_{m}$ peak and the literature value for the enthalpy of purely crystalline PVDF-TrFE $\left(\Delta H_{0}\right)$ was taken as $45 \mathrm{~J} \mathrm{~g}{ }^{-1} .55,56$ For SWCNT/PVDF-TrFE (0.000 wt\% to $\left.0.100 \mathrm{wt} \%\right)$ films, the $\chi_{\mathrm{c}}$ was found to remain relatively unchanged, between $37 \%$ and $41 \%$. At SWCNT concentrations in the films of 
Table 1 Degree of crystallinity $\left(\chi_{c}\right)$ and the relative fractions of the electroactive phase $\left(F_{E A}\right), \beta$-phase $\left(F_{B}\right)$ and $\gamma$-phase $\left(F_{\gamma}\right)$ obtained via DSC, ATR-FTIR spectroscopy, Raman spectroscopy and XRD.

\begin{tabular}{|c|c|c|c|c|c|c|}
\hline & $\chi \mathrm{c}^{\mathrm{a}}(\%)$ & $\mathrm{F}_{E A}{ }^{\mathrm{b}}(\%)$ & $\mathrm{F}_{\beta}^{\mathrm{c}}(\%)$ & $\mathrm{F}_{\beta}{ }^{\mathrm{d}}(\%)$ & $F_{\gamma}{ }^{c}(\%)$ & $\mathrm{Fy}^{\mathrm{d}}(\%)$ \\
\hline PVDF-TrFE & 37.2 & 83.8 & 72.0 & 70.0 & 28.0 & 30.0 \\
\hline 0.005 wt $\%$ SWCNT & 39.7 & 84.3 & 72.5 & 69.2 & 27.5 & 30.8 \\
\hline 0.010 wt\% SWCNT & 40.5 & 83.4 & 72.7 & 69.3 & 27.3 & 30.7 \\
\hline 0.020 wt\% SWCNT & 41.1 & 83.5 & 71.7 & 70.3 & 28.3 & 29.7 \\
\hline 0.050 wt\% SWCNT & 39.0 & 82.9 & 71.6 & 68.7 & 28.4 & 31.3 \\
\hline 0.100 wt\% SWCNT & 41.3 & 81.4 & 64.5 & 70.7 & 35.5 & 29.3 \\
\hline 0.500 wt\% SWCNT & 33.9 & 72.5 & $-^{e}$ & 69.3 & $-^{\mathrm{e}}$ & 30.7 \\
\hline
\end{tabular}

aObtained via DSC; bObtained via ATR-FTIR spectroscopy; cObtained via Raman spectroscopy; dObtained via XRD; eNo polymer peaks were detected due to high SWCNT Raman signal.

0.200 wt $\%$ and above, the $\chi_{c}$ was observed to decrease to between $31 \%$ and $34 \%$.

ATR-FTIR spectroscopy was used to further confirm the phase composition of the extrusion printed SWCNT/PVDF-TrFE (0.000 wt\% to 0.500 wt\%) films, shown in Fig. 2e. The presence of both the $\beta$ phase and $\gamma$ phase of PVDF-TrFE was shown as the combined $\beta+\gamma$ phase peak at $840 \mathrm{~cm}^{-1}$, with separate peaks for the $\beta$ phase at $1285 \mathrm{~cm}^{-1}$ and $\gamma$ phase at $1235 \mathrm{~cm}^{-1} .{ }^{57}$ An additional shoulder at $1430 \mathrm{~cm}^{-1}$ was attributed to the $\beta$ phase. The region commonly ascribed to the $\alpha$ phase at $766 \mathrm{~cm}^{-1}$ showed no peaks, further showing the films consisted of purely piezoelectric polymorphs. ${ }^{58}$

Fig. $2 f$ shows a summary of the percentage phase fractions calculated from the ATR-FTIR spectra (grey), Raman spectra (red) and XRD spectra (blue), with the values presented in Table 1. Using the ATR-FTIR data, the electroactive phase fraction $F_{E A}$, consisting of the $\beta+\gamma$ phases, was calculated from the FTIR spectra using eqn (2).7,57

$$
F_{E A}(\%)=\frac{I_{E A}}{\left(K_{E A} / K_{\alpha}\right) I_{\alpha}+I_{E A}} \times 100
$$

In eqn (2), $I_{E A}$ is the intensity of the IR peak at $840 \mathrm{~cm}^{-1}(\beta+\gamma$ phase), $I_{\alpha}$ is the intensity of the IR peak at $766 \mathrm{~cm}^{-1}$ ( $\alpha$ phase), and $K_{E A}$ and $K_{\alpha}$ are the absorption coefficients for the respective peaks at $840 \mathrm{~cm}^{-1}$ and $766 \mathrm{~cm}^{-1}$, with the values given as $7.7 \times$ $10^{4} \mathrm{~cm}^{2} \mathrm{~mol}^{-1}$ and $6.1 \times 10^{4} \mathrm{~cm}^{2} \mathrm{~mol}^{-1}$, respectively. ${ }^{57,59}$ The values for the $\beta+\gamma$ phase fraction were found to be constant for all measured samples, with a small decrease to $72.5 \%$ at a SWCNT concentration of 0.500 wt\%. No contribution was observed from the $\alpha$ phase of the polymer, showing the extrusion printed films contained primarily both piezoelectric $\beta$ and $y$ phases.

The Raman data was used to calculate the relative fractions of $\beta$ and $\gamma$ phases in the polymer. Commonly, phase analysis in literature consists of the intensity ratio of the $\alpha$ and $\beta$ phase peaks, resulting in a value of zero for pure $\beta$ phase or infinity for pure $\alpha$ phase. ${ }^{53,60}$ To quantify the relative phase content of the $\beta$ and $\gamma$ phases in the SWCNT/PVDF-TrFE (0.000 wt $\%$ to 0.500 wt\%) films, a series of equations were used in this study and are shown in eqn (3) and (4), respectively.

$$
F_{\beta}(\%)=\frac{I_{842}}{I_{842}+I_{811}} \times 100
$$

$$
F_{\gamma}(\%)=\frac{I_{811}}{I_{842}+I_{811}} \times 100
$$

In this form, $I_{842}$ corresponds to the intensity of the $\beta$ phase Raman peak at $842 \mathrm{~cm}^{-1}$ and similarly, $I_{811}$ corresponds to the intensity of the $\gamma$ phase Raman peak at $811 \mathrm{~cm}^{-1} .51,52$ These fractions are presented in red in Fig. 2f. Films with low concentrations of SWCNTs showed similar relative $\beta$ phase fractions compared to pristine PVDF-TrFE films. Conversely, at higher SWCNT concentrations, the $\beta$ phase fraction dropped significantly from the maximum of $72.7 \%$ at 0.010 wt\% SWCNT to a minimum of $48.4 \%$ at $0.200 \mathrm{wt} \%$ SWCNT and the $\gamma$ phase increased from $27.3 \%$ at 0.010 wt $\%$ SWCNT to $51.6 \%$ at 0.200 wt\% SWCNT. Nonetheless, it should be noted that at the higher concentrations, the SWCNT G-band signal was significantly larger than that of the PVDF-TrFE peaks and variations between phases became increasingly difficult to quantify.

The final set of data presented in blue in Fig. $2 f$ was the quantification of phases from the XRD spectra. The peaks at $18.1^{\circ} 2 \theta$ and $20.2^{\circ} 2 \theta$ were deconvoluted, and their relative intensity fractions are shown in Fig. $2 f$ and Table 1 . The PE peak at $18.2^{\circ} 2 \theta$ showed values in line with $F_{\gamma}$ from the Raman analysis and therefore was attributed to the presence of the $\gamma$ phase, although it has been commonly misattributed to the non-polar $\alpha$ phase in previous studies. ${ }^{11,61}$

From the data presented in Fig. 2, it was concluded that minimal changes in phase composition were observed with incorporation of low concentrations of SWCNTs into PVDF-TrFE.

\section{Nanoscale piezoelectric phenomena}

Piezoresponse force microscopy (PFM) was carried out on the samples to further investigate their nanoscale piezoelectric properties. A cantilever with diameter of below $25 \mathrm{~nm}$ was rastered across a $5 \mu \mathrm{m} \times 5 \mu \mathrm{m}$ sample area with voltages applied in a sweeping manner using a pre-defined pattern, shown in Fig. 3a. For each line of scanning, the cantilever swept through the entire range of voltages between $-20 \mathrm{~V}$ and $+20 \mathrm{~V}$ to minimise artefacts arising from the instrument.

Fig. $3 \mathrm{~b}$ shows the corresponding PFM amplitude output after exposing the samples to the range of desired voltages at SWCNT concentrations from $0.000 \mathrm{wt} \%$ to $0.200 \mathrm{wt} \%$ relative to PVDF-TrFE. The amplitude response, corresponding to the piezoelectric output, was found to increase with increasing 


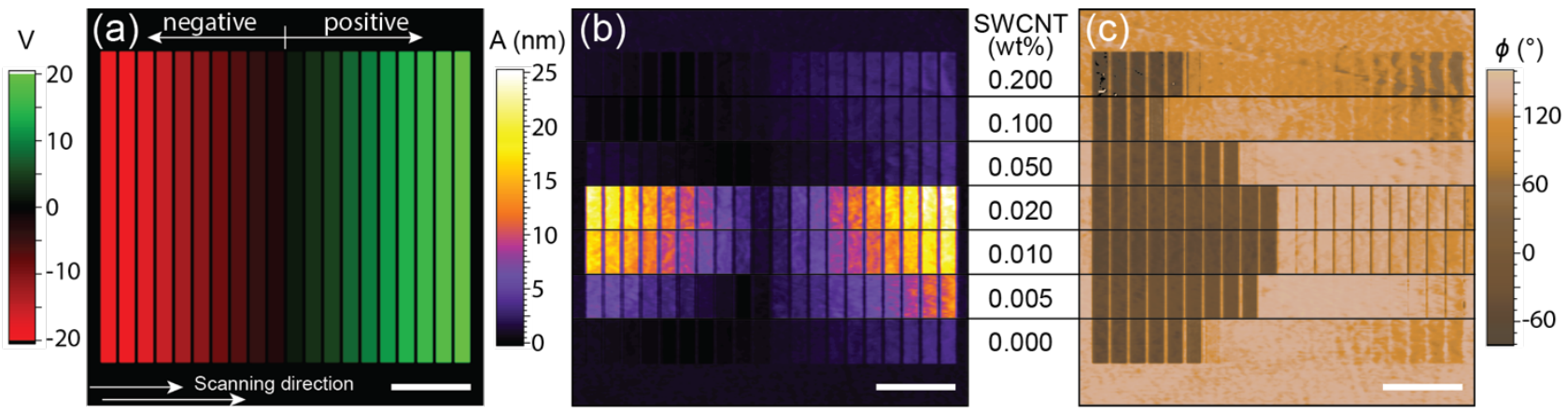

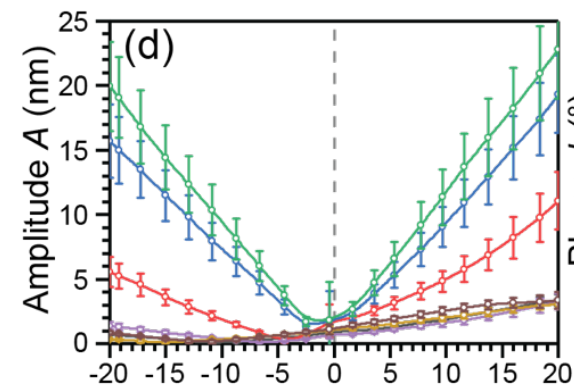

Input voltage (V)
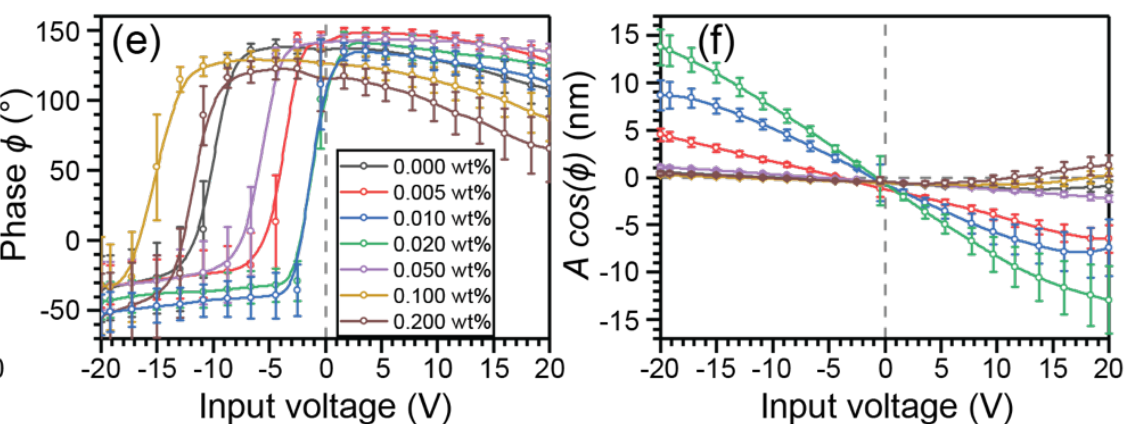

Fig. 3 PFM characterisation of extrusion printed SWCNT/PVDF-TrFE (0.000 wt\% to $0.200 \mathrm{wt} \%)$ films, (a) the pattern of the applied voltage to the cantilever during raster. (b) The piezoelectric amplitude response of the printed samples upon the application of voltage between the cantilever and the substrate and (c) the corresponding PFM phase response, showing representative slices for each sample. (d) The raw amplitude $(A)$. (e) The raw phase $(\phi)$ ). (f) The resultant processed PFM amplitude $(A c o s(\phi))$ as a function of the applied voltage for the investigated SWCNT concentrations. The scale bar represents $1 \mu \mathrm{m}$.

SWCNT concentration up to 0.020 wt\%, showing a 20 -fold increase in amplitude at 0.020 wt\% relative to the PVDF-TrFE sample, exhibiting a sharp decrease at SWCNT concentrations higher than $0.020 \mathrm{wt} \%$. It was evident that while the phase fractions were not significantly altered by the incorporation of SWCNTs (see, Fig. 2 and Table 1), the piezoelectric response showed significantly enhanced properties. Further, the phase switching behaviour, shown in Fig. 3c, was significantly altered by the inclusion of SWCNTs. The switching voltage in this case is proportional to the coercive field $\left(E_{C}\right)$, which is the electric field required to pole the fluoropolymers. At higher SWCNT concentrations (between 0.100 wt\% and 0.200 wt\%), the switching voltage between $-14 \mathrm{~V}$ and $-10 \mathrm{~V}$ was comparable to the pristine PVDF-TrFE film; however, at lower SWCNT concentrations (between $0.010 \mathrm{wt} \%$ and $0.020 \mathrm{wt} \%$ ) the switching voltage dropped to between $-2 \mathrm{~V}$ and $0 \mathrm{~V}$. A switching voltage below $2 \mathrm{~V}$ corresponds to an electric field below $50 \mathrm{kV}$ $\mathrm{m}^{-1}$, which is orders of magnitude lower than any previous reported values for electrical poling of fluoropolymers (typically $4 \mathrm{MV} \mathrm{m}^{-1}$ to $500 \mathrm{MV} \mathrm{m}^{-1}$ ). ${ }^{7,9,19}$

The amplitude and phase were then extracted from the PFM data as a function of the input voltage and are presented in Fig. $3 \mathrm{~d}$ and Fig. 3e, respectively. The amplitude was shown to scale linearly with the input voltage over the range of $-20 \mathrm{~V}$ and +20 V. The amplitude minima were attributed to the dipole orientation switching of the PVDF-TrFE. The phase was found to be maximised in the direction of the positive voltage, even when no voltage was applied, for all samples excluding those containing SWCNT concentrations of $0.010 \mathrm{wt} \%$ and $0.020 \mathrm{wt} \%$. Further increase in phase were found for $0.010 \mathrm{wt} \%$ and 0.020 wt\% SWCNT/PVDF-TrFE films upon the application of $+2 \mathrm{~V}$.
Additionally, the phase angle of the $0.200 \mathrm{wt} \%$ SWCNT/PVDFTrFE film was found to degrade at positive applied voltages, with a maximum at $-2 \mathrm{~V}$.

The effective $d_{33}$ coefficient can be obtained from the PFM data using eqn (5). ${ }^{62}$

$$
d_{33}=\frac{A \cos (\phi)}{Q V}
$$

Here, $A$ represents the PFM amplitude in units of $p m, \phi$ represents the PFM phase angle, $Q$ represents the unitless quality factor of the cantilever resonance peak obtained during the PFM experiments, which in this case was consistent between 70 and 92 with slight variations between samples, and $V$ represents the applied voltage. For the analysis of the data, individual pixels at each position and corresponding to their respective voltages were used for the calculation and an average was taken for the entire set of pixels corresponding to the respective applied voltage.

Fig. $3 f$ shows the $A \cos (\phi)$ plotted as a function of the applied voltage for the extrusion printed SWCNT/PVDF-TrFE (0.000 wt\% to 0.200 wt\%) films investigated during this experiment. Typically in literature, a linear trend is observed at low applied voltages, with a decreasing slope as the films approach the maximum induced strain. All of the investigated films showed trends following literature for fluoropolymers. Degradation was observed at high magnitudes of applied voltage in the positive direction for 0.000 wt\%, $0.100 \mathrm{wt} \%$ and $0.200 \mathrm{wt} \%$ SWCNT/PVDF-TrFE films, as the $E_{C}$ values for these samples were below $-10 \mathrm{~V}$. Therefore, a moderate voltage of $+10 \mathrm{~V}$ was chosen for the characterisation of the $d_{33}$ coefficient, corresponding to approximately $0.25 \mathrm{MV} \mathrm{m}^{-1}$ and well below the typical values for $E_{C}$ for fluoropolymers, while additionally 
probing in the direction the films were polarised during deposition.

The extrusion printed PVDF-TrFE (0.000 wt\% SWCNT) film showed a low response under voltage $(+10 \mathrm{~V})$, suggesting low relative polarisation with a $d_{33}$ coefficient of $-1.9 \mathrm{pm} \mathrm{V}^{-1}$ at +10 $\checkmark$ (Fig. S5). A moderate increase in SWCNT concentration to 0.005 wt\% resulted in a sharp increase of the amplitude and therefore the $d_{33}$ coefficient to $-5.9 \mathrm{pm} \mathrm{V}^{-1}$, corresponding to a $210 \%$ enhancement. The maximum $d_{33}$ was found at 0.020 wt $\%$ SWCNT, at $-12 \mathrm{pm} \mathrm{V}^{-1}$, a $500 \%$ improvement over that of the pristine PVDF-TrFE films. This $d_{33}$ value is comparable to work by Bodkhe et al. ${ }^{45}\left(d_{31}\right.$ at $\left.18 \mathrm{pm} \mathrm{V}^{-1}\right)$ for BTO/PVDF composite structures and commercially available piezoelectric PVDF $\left(d_{33}\right.$ at $-33 \mathrm{pm} \mathrm{V}-1$, PFM results shown in Fig. S6, ESI+) for mechanically drawn and poled films. ${ }^{20}$ Further increases in SWCNT concentration resulted in a reduction back to a lower $d_{33}$ coefficient of $-1.7 \mathrm{pm} \mathrm{V}^{-1}$ (0.050 wt\% SWCNTs), similar to that of the pristine PVDF-TrFE (0.000 wt\% SWCNTs) films. A further decrease in the $d_{33}$ coefficient at 0.100 wt\% and 0.200 wt\% SWCNTs was also observed.

In literature, increases in energy harvesting efficiencies of nanocomposite fluoropolymer PEGs have been largely attributed to enhancements in crystallinity and relative $\beta$ phase fractions within the polymer. Here, we see no crystallinity or relative $\beta$ phase fraction with increased SWCNT content in the PVDF-TrFE (see Fig. 2 and Table 1); however, we do see a marked increase in the polarisation (Fig. 3). This result demonstrates that maximising the net polarisation vector is a key consideration for optimising piezoelectric energy harvesting and can be achieved by either aligning the local dipoles or enhancing the relative $\beta$ phase fraction. The incorporation of SWCNTs into fluoropolymers at low concentrations, as shown via PFM (Fig. 3) and polymer phase composition analysis (Fig. 2), does not result in an increase in $\beta$ phase, and thus the improvement in net polarisation must arise from dipole alignment.

\section{Macroscale piezoelectric phenomena}

To further explore the piezoelectric energy harvesting properties of the extrusion printed SWCNT/PVDF-TrFE films, PEGs were fabricated by coating the films with $\mathrm{Cr} / \mathrm{Au}$ electrodes, attaching external wires via conductive $\mathrm{Cu}$ foil adhesive and packaging with insulating polyimide tape. Energy harvesting experiments were undertaken through cyclic compression of the PEGs to investigate the correlation between the phase properties, nanoscale piezoelectric phenomena and bulk energy conversion capabilities.

Fig. 4a shows the generated voltage traces under $5 \mathrm{~Hz}$ cyclic compression of the PEGs for the extrusion printed SWCNT/PVDF-TrFE (0.000 wt\% to 0.500 wt\%) PEGs, reported using an oscilloscope with a $10 \mathrm{M} \Omega$ input impedance. Here, similar trends were observed to the PFM data (see Fig. S7, ESI + ). The data for the pristine PVDF-TrFE (0.000 wt\% SWCNTs) PEG showed the presence of electrical output arising from shearinduced alignment during extrusion printing, however the magnitude was found to be low. When the shear alignment from extrusion printing was coupled with the incorporation of SWCNTs, the generated peak-to-peak voltage $\left(V_{p p}\right)$ trend was in close agreement with the $d_{33}$ values obtained from PFM. The $V_{p p}$ was found to increase with increases in SWCNT concentration of the PEGs up to a maximum at $0.020 \mathrm{wt} \%$ SWCNT. At SWCNT concentrations above $0.020 \mathrm{wt} \%$, decreases in $V_{p p}$ were observed, with values similar to the pristine PVDF-TrFE $(0.000$ wt\% SWCNTs) PEGs. The sample containing 0.500 wt\% SWCNT did not exhibit any voltage output from cyclic compression, which was attributed to conductive SWCNT networks forming between the electrodes in the film. This was supported by HIM imaging (Fig. 1) and confirmed using cyclic voltammetry, shown in Fig. S8 (ESI $)$ ), whereby the 0.500 wt\% SWCNT/PVDF-TrFE PEG exhibited conductive behaviour.

Given the maximum $V_{p p}$, the energy harvesting characteristics of extrusion printed SWCNT/PVDF-TrFE $(0.020$ wt\%) PEG were investigated further. The output voltage was measured under cyclic compression at $5 \mathrm{~Hz}$ with the load

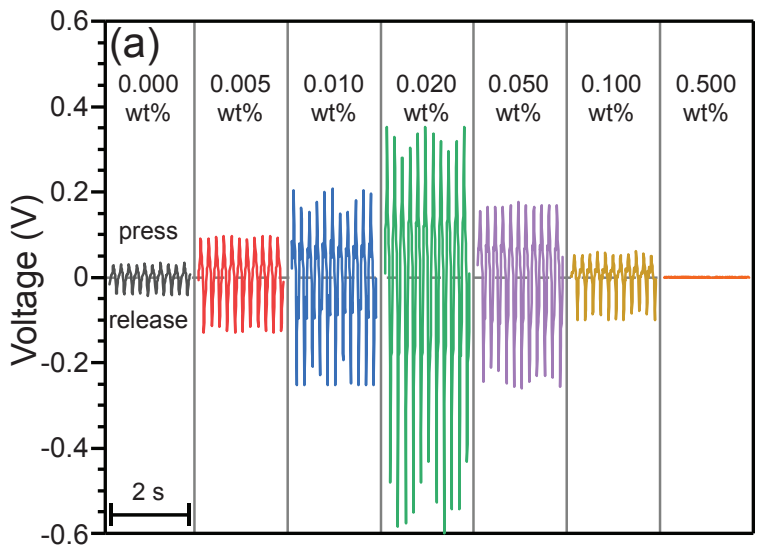

Time (s)

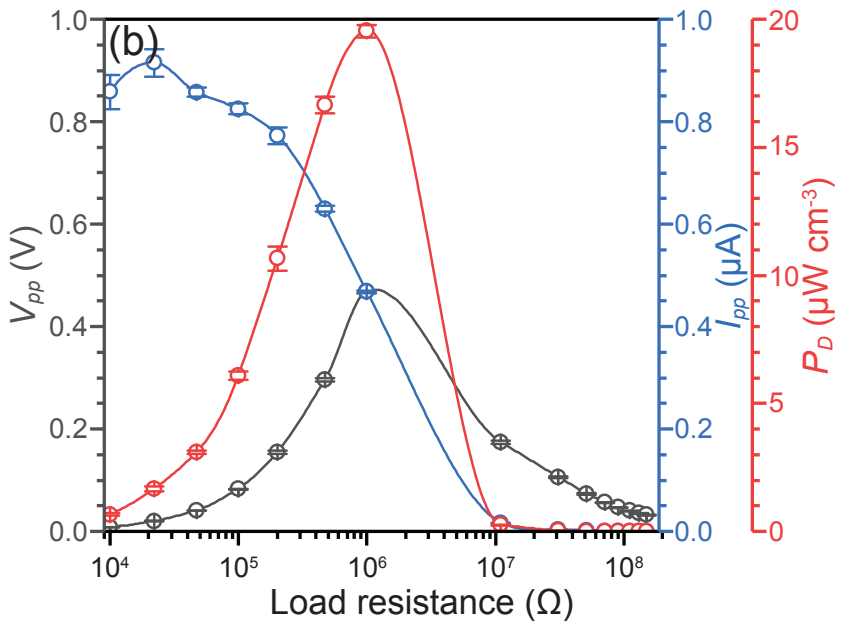

Fig. 4 Energy generation characteristics of the extrusion printed SWCNT/PVDF-TrFE (0.000 wt \% to 0.500 wt\%) PEGs, showing (a) the output voltage as a function of time arising from imparted cyclic compression of the samples at $5 \mathrm{~Hz}$ for the SWCNT concentrations between $0.000 \mathrm{wt} \%$ and $0.500 \mathrm{wt} \%$ using an Instron testing machine at a controlled strain rate. (b) Peak to peak voltage (Vpp) (grey), peak to peak current (Ipp) (red) and power density (PD) (blue) for the 3D printed SWCNT/PVDF-TrFE (0.020 wt\%) $\mathrm{PEG}$ as a function of the load resistance. The input impedance of measurement for the tests was $10 \mathrm{M} \Omega$. 
resistor placed in parallel with the PEG, for resistances between $1 \mathrm{k} \Omega$ and $130 \mathrm{M} \Omega$, with the $V_{p p}$ values shown in Fig. $4 \mathrm{~b}$ in grey. The voltage signal was shown to increase up to $1 \mathrm{M} \Omega$, and at 10 $\mathrm{M} \Omega$ began to decrease due to the load resistance reaching the input impedance of the measuring system and therefore presenting voltage values lower than expected. Furthermore, it was noted that the voltage at $1 \mathrm{M} \Omega$ was lower relative to the measurement with no load resistance (shown in Fig. 4a), suggesting the maximum $P_{D}$ could occur at load resistances between $1 \mathrm{M} \Omega$ and $10 \mathrm{M} \Omega$. The current output was calculated using $I=V / R$ and showed close agreement in the trend to previous reports, with a maximum generated current of $0.9 \mu \mathrm{A}$, corresponding to the short-circuit current $\left(I_{s c}\right)$ at the plateau for load resistance below $10^{5} \Omega$. The $I_{s c}$ for the SWCNT/PVDF-TrFE (0.020 wt\%) PEG corresponds closely to previous work on other self-poled fluoropolymer-based energy harvesters. ${ }^{37,63-70}$

The volumetric power density $P_{D}$ was then calculated for the extrusion printed SWCNT/PVDF-TrFE (0.020 wt\%) PEG using eqn (6) and shown in Fig. $4 \mathrm{~b}$ as a function of load resistance.

$$
P_{D}=\frac{V_{p p}^{2}}{R\left(t \times A_{e}\right)}
$$

In eqn (6), $R$ represents the load resistance, $t$ represents the sample thickness (shown in Fig. S9 and Fig. S10, ESIt) and $A_{e}$ represents the active electrode area overlapping on both surfaces of the film, taken as $2.1 \mathrm{~cm}^{2}$. The maximum $P_{D}$ for the SWCNT/PVDF-TrFE (0.020 wt\%) PEG was found to be $19.8 \mu \mathrm{W}$ $\mathrm{cm}^{-3}$ at $1 \mathrm{M} \Omega$ load resistance (shown in Fig. $4 \mathrm{~b}$ in blue, and in Fig. 5 as a solid red diamond). The comparison to literature values for the $P_{D}$ of PEGs incorporating CNTs is shown in Fig. 5 as a function of CNT concentration. PEGs poled via electric fields are shown as circles and unpoled PEGs are shown as diamonds. The data is further expanded upon in Table S1 (ESI $)$ ).

Comparatively, Lee et al. ${ }^{39}$ have reported on unpoled MWCNT/PVDF composites at MWCNT concentrations up to 0.075 wt $\%$. They observed enhancements in energy harvesting (maximum $1.2 \mathrm{~V}$ and $3.8 \mathrm{nA}$ for $0.075 \mathrm{wt} \% \mathrm{MWCNT}$ ) without

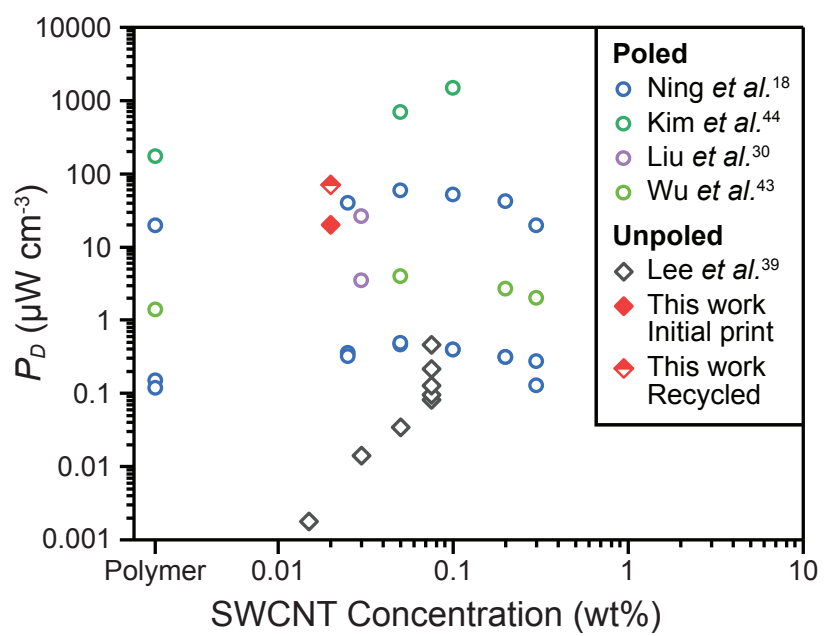

Fig. 5 Volumetric power density PD for PEGs containing CNTs as a function of CNT concentration for this study and previous publications reporting energy harvesting capabilities. Circles represent electrically poled PEGs and diamonds represent unpoled PEGs. Fluoropolymer PEGs without CNTs are presented where found in the literature for comparison. using electrical poling (Fig. 5, grey diamonds). Few details were given on the device characteristics and measurement conditions, therefore the device thickness was assumed to be $100 \mu \mathrm{m}$ to obtain $P_{D}$ values, which were calculated as $0.46 \mu \mathrm{W}$ $\mathrm{cm}^{-3}$. In comparison, the value for maximum resistancematched $P_{D}$ in this study of $19.8 \mu \mathrm{W} \mathrm{cm}{ }^{-3}$ is greater than one order of magnitude higher than that of Lee et al. ${ }^{39}$

The $P_{D}$ values from this work fall well within the range of PEGs consisting of CNTs and fluoropolymers, obtained through mechanical drawing at elevated temperatures and electrical poling, namely that of Ning et al. ${ }^{18}$ (poling electric field at $60 \mathrm{MV}$ $\mathrm{m}^{-1}$ ) and Liu et al. ${ }^{30}\left(7 \mathrm{MV} \mathrm{m}^{-1}\right)$ (Fig. 5).

\section{Computational analysis of behaviour at the interface between SWCNT and PVDF-TrFE}

To further investigate the atomic-scale interfacial phenomena between SWCNTS and PVDF-TrFE, and confirm the enhancements in polarisation arising from SWCNT incorporation, all-atom Molecular Dynamics (MD) simulations were employed. Two SWCNT chiralities were analysed, namely the armchair $(12,0)$ and the zig-zag $(8,8)$, corresponding to semiconducting and conducting SWCNTs, respectively.

Fig. 6a shows the probability distribution of the angle $\varphi$, $P(\varphi)$, formed between the SWCNT axis of symmetry vector and the SWCNT/PVDF-TrFE dipole moment vector for the $(8,8)$ (black line) and the $(12,0)$ (red line) SWCNT configurations. The representative simulation configurations are shown in the inset in Fig. 6a. The results were averaged over the simulation time for three independent simulation iterations in order to obtain statistical significance. The shaded regions in Fig. 6a-c represent the error bar obtained from three different MD simulations. For $\varphi=0^{\circ}$ and $180^{\circ}$, the dipole moment vector of the SWCNT/PVDFTrFE system was found to be either parallel or antiparallel to the SWCNT axis of symmetry. For both SWCNT configurations, the probability of forming angles of $\varphi=90^{\circ}$ (dipole moment vector perpendicular to the SWCNT) was small. Initially ( $t=0 \mathrm{~ns}$ ), the two PVDF-TrFE chains were placed randomly away from each other and close to the SWCNT (Fig. 6d, top snapshots) with initial $\varphi$ angle almost perpendicular to the SWCNT axis of symmetry. For both SWCNT configurations, however, and once the copolymers coiled on the SWCNT surface, the probability of forming angles of $\varphi=90^{\circ}$ (dipole moment vector perpendicular to the CNT) was small. The interactions between the SWCNT and the PVDF-TrFE caused the latter to fold around the SWCNT (first frames of Video S1), aligning their dipole moment vector immediately with the SWCNT backbone. The PVDF-TrFE chains have flexible backbones, coiling around each other and exhibiting little or no wrapping around the SWCNT. In fact, the dipole moment vector of the SWCNT/PVDF-TrFE was found to be more likely to form small angles $\left(0^{\circ}\right.$ to $45^{\circ}$ or $135^{\circ}$ to $\left.180^{\circ}\right)$ with the SWCNT axis of symmetry, especially when arranged in the $(12,0)$ SWCNT.The copolymer chains had higher interaction energy with the $(12,0)$ SWCNT than with the $(8,8)$ SWCNT, indicating that the SWCNT chirality affects the alignment of the dipole moment vector with the SWCNT backbone, as both SWCNT types investigated here had comparable radii. 

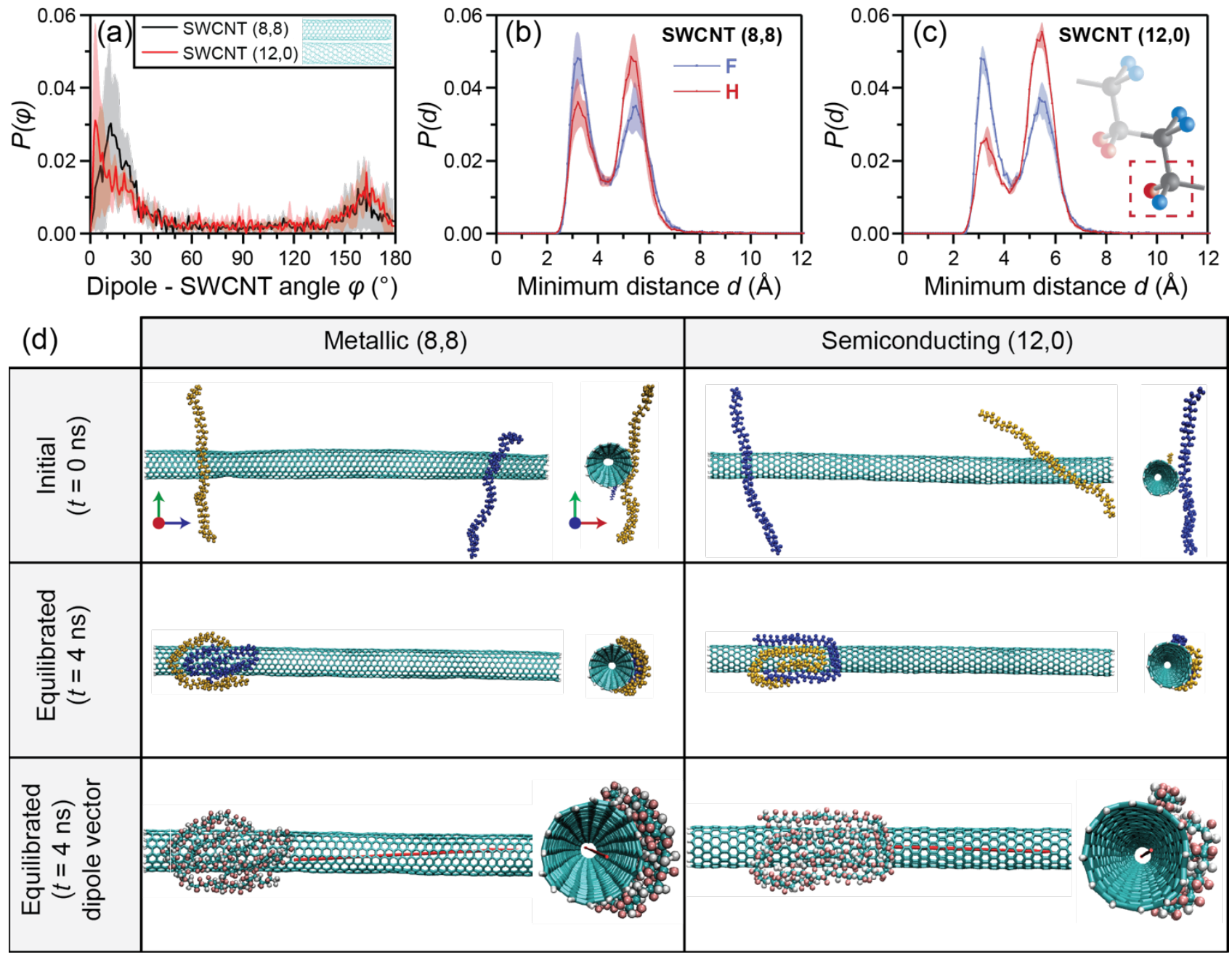

Fig. 6 (a) The probability distribution of the angle between the vectors of the SWCNT axis of symmetry and the SWCNT/PVDF-TrFE dipole moment for the (8,8) (black line) and the $(12,0)$ (red line) SWCNT configurations (insets). The probability distribution of the minimum distance, d, between fluoride (blue lines) or hydrogen atoms (red lines) of the TrFE units (inset of a faded VDF and a highlighted TrFE monomer) and the CNT with (b) $(8,8)$ and $(c)(12,0)$ chirality. The shaded regions represent the error bar obtained from three independent MD simulations. (d) Representative initial spatial arrangement at 0 ns (top) of two PVDF-TrFE chains (gold and blue) relative to SWCNTs (cyan) with (8,8) (left) and (12,0) (right) chiralities, the spatial arrangement after equilibration at $4 \mathrm{~ns}$ (middle) and the representative PVDF-TrFE dipole moment vector direction after equilibration at $4 \mathrm{~ns}$ (bottom).

Furthermore, MD simulations with one PVDF-TrFE co-polymer chain exhibited slightly larger $\varphi$ angles than those of the system with two co-polymer chains for the $(8,8)$ SWCNT, revealing that steric effects between the chains affect the direction of the dipole moment vector.Videos showing the temporal evolution of the dipole moment vector of the SWCNT/PVDF-TrFE system, as well as the dipole moment vectors of each of the two PVDFTrFE chains, are presented in Video S1 (ESIt).

The probability distribution of the minimum distance, $d$, of fluoride (blue) and hydrogen atoms (red) of the TrFE units within the PVDF-TrFE chains from the SWCNT with $(8,8)$ and $(12,0)$ chiralities is shown in Fig. $6 \mathrm{~b}$ and $6 \mathrm{c}$. The minimum distance is calculated only for the $\mathrm{H}-\mathrm{C}-\mathrm{F}$ branch of the TrFE units (highlighted unit in the inset of Fig. 6b) of the PVDF-TrFE chains as the branches of the VDF units are symmetric with either two $\mathrm{H}$ atoms $(\mathrm{H}-\mathrm{C}-\mathrm{H})$ of two $\mathrm{F}$ atoms $(\mathrm{F}-\mathrm{C}-\mathrm{F})$. The shaded regions in Fig. 7 correspond to the error obtained from the three MD simulations. The results were averaged over all the TrFE units within the two co-polymer chains for the entire simulation time. The $\mathrm{C}$ and $\mathrm{F}$ atoms of the TrFE $\mathrm{H}-\mathrm{C}-\mathrm{F}$ branch were found to be most probable in two distinct regions: at approximately $3.5 \AA$ and $5.5 \AA$ away from the SWCNT surface. However, the $\mathrm{F}$ atoms were more likely to be arranged closer to the SWCNT surface than the $\mathrm{H}$ atoms, as indicated by their higher probability distribution at $3.5 \AA$. This preference of fluoride to remain closer to the SWCNT surface was even more pronounced when the PVDF-TrFE chains interact with the zigzag $(12,0)$ SWCNTs, revealing larger differences in their probability distributions compared to the $\mathrm{H}$ atoms. The $\mathrm{H}$ and $\mathrm{F}$ arrangement with respect to the SWCNT surface hardly changes during the simulations indicated by the narrow error bars in their probability distributions. Furthermore, the $\mathrm{H}-\mathrm{C}$ and the $\mathrm{C}$ - $\mathrm{F}$ bonds of the TrFE $\mathrm{H}-\mathrm{C}-\mathrm{F}$ branch arrange perpendicularly to the CNT surface as shown by the probability distribution of their angle with the CNT axis of symmetry with a peak at $90^{\circ}$ (Fig. S11, ESIt).

The PVDF-TrFE chain was initially placed tangential to the SWCNT surface, perpendicular to the SWCNT length axis (Fig. 
6c). Rapidly, the PVDF-TrFE was found to adsorb to the SWCNT surface, indicating the attractive interaction between the polymer and the SWCNT. The orientation of the dipoles within PVDF-TrFE, as shown in Fig. 6a, showed preferential alignment toward low angles relative to the SWCNT length axis, with values between $0^{\circ}$ and $30^{\circ}$, as well as $135^{\circ}$ and $180^{\circ}$, confirming the presence of preferential alignment of the dipole to the SWCNT within the extrusion printed polymer. Additionally, the fluorine-hydrogen dipole vector within the $\mathrm{H}-\mathrm{C}-\mathrm{F}$ group was found to preferentially orient away from the SWCNT when simulating a single PVDF-TrFE chain on the SWCNT (Fig. 6b and 6c).

The MD simulations were in close agreement with the nanoscale PFM data presented in Fig. 3, which showed a fivefold increase in polarisation upon the incorporation of low (0.020 wt\%) concentrations of SWCNTs. At concentrations above $0.020 \mathrm{wt} \%$, however, the SWCNTs reached a concentration stability threshold with an increased tendency to aggregate and therefore exhibited a decrease in the polarisation. Similar results were found in the macroscale energy harvesting experiments (Fig. 4). The maximum energy conversion was found to occur in the SWCNT/PVDF-TrFE $(0.020$ wt\%) PEGs, subsequently showing a decreased templating effect at higher SWCNT concentrations. For SWCNT/PVDF-TrFE (0.100 wt\%) PEGs, the output voltage under cyclic compression was found to be comparable to the SWCNT/PVDF-TrFE $(0.000$ wt\%) PEGs. Furthermore, at a SWCNT concentration of 0.500 $w t \%$, the SWCNTs formed a conductive pathway (Fig. S5, ESIt), crossing the percolation threshold and therefore showing no energy harvesting characteristics (Fig. 4).

These MD calculations have shown conclusively that the SWCNTs can template the PDVF-TrFE molecules inducing dipole alignment around $\mathrm{a} \pm 45^{\circ}$ arc relative to the SWCNT backbone. This alignment, however, is only expected to occur when there are non-bundled SWCNTs and thus, at higher mass loadings where aggregated SWCNTs dominate the material properties, a sharp reduction in energy harvesting outputs was observed. In contrast to previous literature of CNT/PVDF-TrFE composites, the use of SWCNTs in this work enabled a consistent dipole alignment as the electrons are localised around a single CNT layer. In the literature, which focuses on MWCNTs, this alignment effect for inducing preferential polarisation is expected to be significantly lower. The concentric structure of the MWCNTs reduces the ability for PVDF-TrFE to influence the total electron density and therefore not produce the dipole alignment effect observed in SWCNTs. It is inferred that this effect was similarly observed in poled fluoropolymers, by Kim et al. ${ }^{44}$, showing a significantly higher $\mathrm{P}_{\mathrm{D}}$ when incorporating SWCNTs than any reported MWCNT/fluoropolymer systems such as Ning et al. ${ }^{18}$. Based on this fundamental understanding, the combination of the dipole alignment via SWCNT templating with partial dipole alignment of the polymers and SWCNTs through extrusion printing, enables this work to achieve power densities and peak to peak voltages comparable to electrically poled PVDF.

\section{Recyclability of extrusion printed films}

The recent review presented by Stadlober et al. ${ }^{71}$ discussed the need for finding recyclable materials for energy harvesting. Tamang et al. ${ }^{72}$ proposed a route to biodegradable PEGs through the introduction of DNA into PVDF, however the publication lacked data to support the claim of biodegradation characteristics. Fluoropolymers, owing to their inherent chemically inert nature, as well as thermal stability, have seen few reports on degradation and recycling in the past. A key aspect towards the commercial applicability of recyclable PEGs is the utilisation of green solvents and quick timeframe of the recycling procedure.

Fig. 7 shows the capability of the extrusion printed SWCNT/PVDF-TrFE (0.020 wt\%) films (Fig. 7a), with the highest polarisation templating effect, to be recycled and reprinted for further use. The initial extrusion printed films (acetone and DMF, 60:40 vol\%) exhibited complete dissolution in pure acetone, classified as a green solvent, in less than $20 \mathrm{~s}$ (Fig. 7a-c and Video S2, ESI ${ }^{7}{ }^{73,74}$ Fig. $7 \mathrm{~b}$ and $7 \mathrm{c}$ show the sample dissolution at $2 \mathrm{~s}$ after initial immersion and $9 \mathrm{~s}$ after initial immersion, respectively ( 0.200 wt\% SWCNT/PVDF-TrFE shown for visual clarity). For reprinting, four initial extrusion printed SWCNT/PVDF-TrFE (0.020 wt\%) films weighing $214 \mathrm{mg}$ were dissolved in $904 \mu \mathrm{L}$ acetone, corresponding to a polymer concentration of $23 \mathrm{wt} \%$.

After the dissolution of the SWCNT/PVDF-TrFE (0.020 wt\%) films (extrusion printed from acetone and DMF, 60:40 vol\%) in pure acetone, they were reprinted directly from acetone without any additional pre-processing. The printing was undertaken at extremely low extrusion pressures relative to the initial print, decreasing by $82.3 \%$ to 0.3 Bar. Furthermore, the lower solvent boiling point noticeably decreased the solvent evaporation time within the printed film. Fig. $7 d$ shows an optical image of the film reprinted directly from acetone. The recycled films exhibited transparency and flexibility similar to the films prior to recycling with no visible aggregation of SWCNTs. The comparative HIM micrographs are shown in Fig. 7e for the SWCNT/PVDF-TrFE (0.020 wt\%) film printed from acetone and DMF mixture (Fig. 7e, top) and the film recycled from acetone (Fig. 7e, bottom). The recycled SWCNT/PVDF-TrFE (0.020 wt\%) film showed minimal SWCNT aggregation, with only slightly higher affinity to bundle, which was attributed to the lack of sonication.

Interestingly, the $\gamma$ and $\beta$ phase ratios appeared to remain relatively unchanged after recycling and reprinting. The Raman analysis showed that the recycled films had comparable phase ratios after reprinting with respect to the $\gamma$ and $\beta$ phase peak ratios at $811 \mathrm{~cm}^{-1}$ and $842 \mathrm{~cm}^{-1}$, respectively (Fig. S12, ESI + ). Furthermore, the XRD analysis (Fig. S13, ESI + ) showed similar $\beta$ and $\gamma$ phase peaks to the initial extrusion printed SWCNT/PVDFTrFE (0.020 wt\%) films.

The nanoscale piezoelectricity was probed with the piezoelectric amplitude shown as a comparison to the initial extrusion printed SWCNT/PVDF-TrFE (0.020 wt\%) film in Fig. $7 f$. The amplitude showed good agreement between the film prior to recycling and post-recycling, confirming the recycled film 

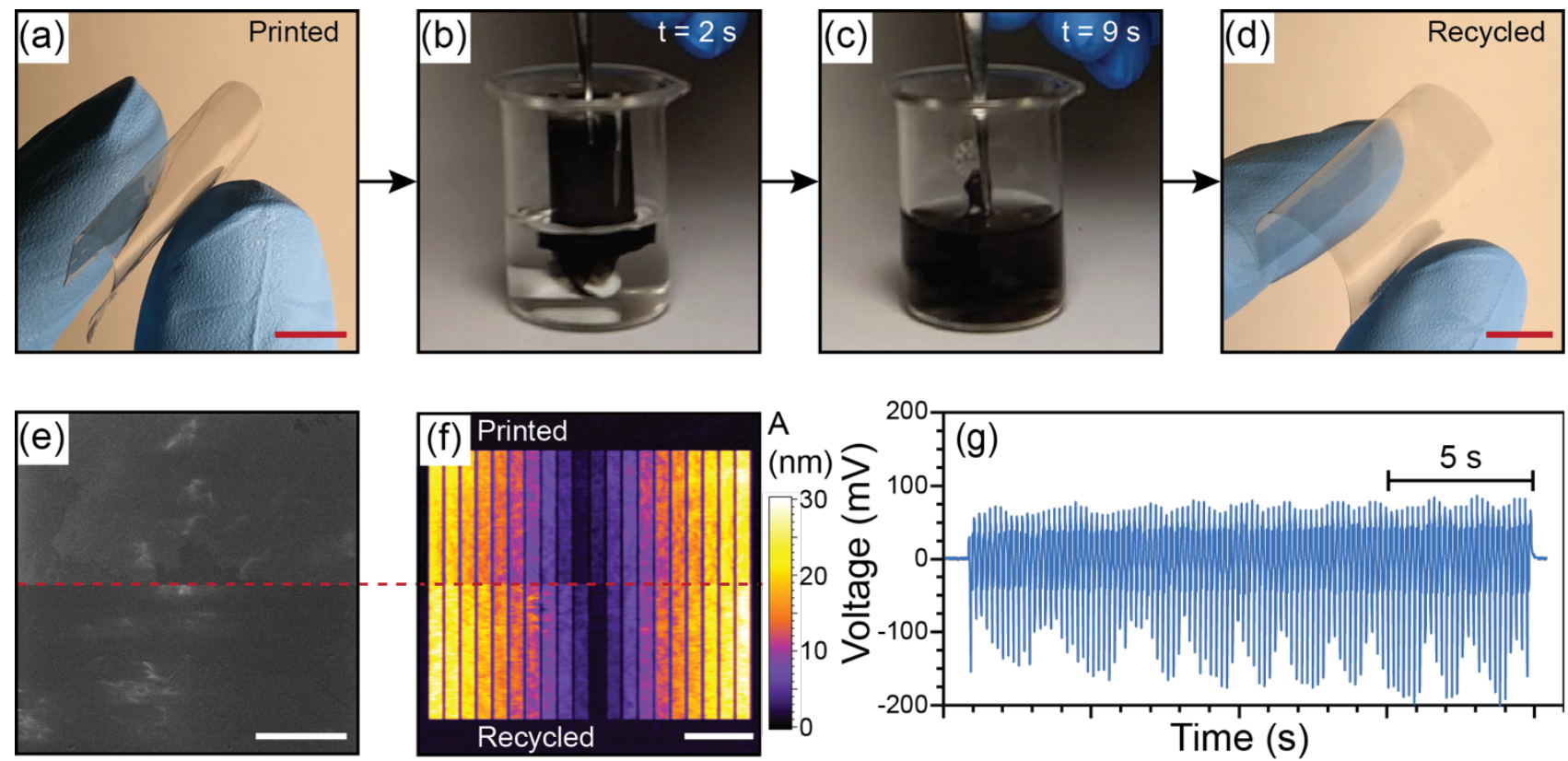

Fig. 7 Recyclability of SWCNT/PVDF-TrFE films (extrusion printed from acetone and DMF, 60:40 vol\%) in pure acetone, with (a) optical image demonstrating the transparency and mechanical flexibility of the initial extrusion printed SWCNT/PVDF-TrFE (0.020 wt\%) film. (b) After $2 \mathrm{~s}$ of immersion in pure acetone. (c) After $9 \mathrm{~s}$ immersion in pure acetone. (d) SWCNT/PVDF-TrFE (0.020 wt\%) film after recycling and reprinting from pure acetone. The 0.200 wt\% sample is shown in (b) and (c) for visual clarity. (e) Top, HIM micrograph of SWCNT/PVDF-TrFE (0.020 wt\%) film printed from acetone/DMF. Bottom, recycled and reprinted film from pure acetone. (f) Top, PFM amplitude trace after application of voltage to the SWCNT/PVDF-TrFE (0.020 wt\%) film printed from acetone/DMF. Bottom, recycled and reprinted film. (g) Voltage output under cyclic compression for a recycled and reprinted SWCNT/PVDF-TrFE (0.020 wt\%) PEG, obtained with an input impedance at $10 \mathrm{M} \Omega$. Scale bars in (a) and (d) correspond to $10 \mathrm{~mm}$, scale bar in (e) corresponds to $10 \mu \mathrm{m}$ and scale bar in (f) corresponds to $1 \mu \mathrm{m}$.

retained its piezoelectric activity. The $d_{33}$ coefficient value for the recycled and reprinted film was found to be $-10 \mathrm{pm} \mathrm{V}^{-1}$ (Fig. $\mathrm{S} 5, \mathrm{ESI}+)$, which was slightly lower than that of the films printed from acetone and DMF $\left(-12 \mathrm{pm} \mathrm{V}^{-1}\right)$.

The recycled SWCNT/PVDF-TrFE (0.020 wt\%) films were then used to fabricate PEGs. The final devices were tested under cyclic compression to demonstrate the retention of energy harvesting after recycling. The voltage is shown as a function of time for $5 \mathrm{~Hz}$ compression in Fig. 7g. The PEG, recycled from a printed SWCNT/PVDF-TrFE (0.020 wt\%) film using pure acetone without any highly energetic processing techniques, was found to produce an average $V_{p p}$ of $209 \mathrm{mV}$ under controlled strain rate at $10 \mathrm{M} \Omega$ input impedance, corresponding to a $P_{D}$ of 0.5 $\mu \mathrm{W} \mathrm{cm} \mathrm{cm}^{-3}$.

The recycled SWCNT/PVDF-TrFE (0.020 wt\%) PEG was then tested using a technique most commonly found in literature, namely cyclic compression under low force using finger pinching. ${ }^{75,76}$ In this case, a $V_{p p}$ of $1.6 \mathrm{~V}$ was obtained at an input impedance of $10 \mathrm{M} \Omega$ (Fig. 8a). The maximum $V_{p p}$ for the recycled 0.020 wt\% SWCNT/PVDF-TrFE PEG was $2.0 \mathrm{~V}$, obtained at an input impedance of $20 \mathrm{M} \Omega$ representing open-circuit voltage conditions (Fig. $8 \mathrm{~b}$ ). The maximum $V_{p p}$ was in close agreement with that of a PEG fabricated using commercial poled PVDF film obtained from Measurement Specialities (Fig. S14, ESIt). The maximum $I_{p p}$ of the recycled $0.020 \mathrm{wt} \%$ SWCNT/PVDF-TrFE PEG was calculated to be $1.2 \mu \mathrm{A}$ at $100 \mathrm{k} \Omega$, corresponding to the short-circuit current (Fig. 8c). The maximum $P_{D}$ for the recycled PEG, obtained under the low-force compression through load resistance matching experiments (Fig. 8c), was $70.6 \mu \mathrm{W} \mathrm{cm}-3$ at $5 \mathrm{M} \Omega$, significantly higher than that of the controlled strain rate experiments. A maximum $P_{D}$ of $70.6 \mu \mathrm{W} \quad \mathrm{cm}^{-3}$ places these recycled and reprinted SWCNT/PVDF-TrFE (0.020 wt\%) PEGs in line with that of poled CNT-fluoropolymer composite PEGs. In fact, they are far higher

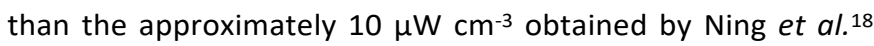
and thus show an incredible promise for future applications in this area. When the PEG was compressed and held prior to release, only a positive peak was observed, with the negative peak following the release, as shown in Fig. 8e, confirming the energy harvesting signal was occurring due to piezoelectricity.

The recycled $0.020 \mathrm{wt} \%$ SWCNT/PVDF-TrFE PEG was further subjected to extensive cycling study, shown in Fig. 8f. The data exhibited no notable decrease in energy harvesting capability after approximately 10000 cycles, confirming long-term cycling stability of the extrusion printed SWCNT/PVDF-TrFE PEGs. Additionally, to demonstrate the potential to capture and store this energy for future use, charging $0.1 \mu \mathrm{F}, 1 \mu \mathrm{F}$ and $10 \mu \mathrm{F}$ capacitors though the simple application of a finger press on the piezoelectric. These capacitors were charged through a bridge rectifier to $3.5 \mathrm{~V}$ over 1.2 minutes, 4.5 minutes, and 24.3 minutes, respectively, with a linear charging profile for the 0.1 $\mu \mathrm{F}$ and $1 \mu \mathrm{F}$ capacitor. The self-poled, recycled, $0.020 \mathrm{wt} \%$ SWCNT/PVDF-TrFE PEG charged these capacitors faster than state-of-the-art piezoelectric polymer PEGs in the literature, demonstrating the feasibility of using these devices to capture human motion to power remote and portable electronics. ${ }^{77,78}$ 


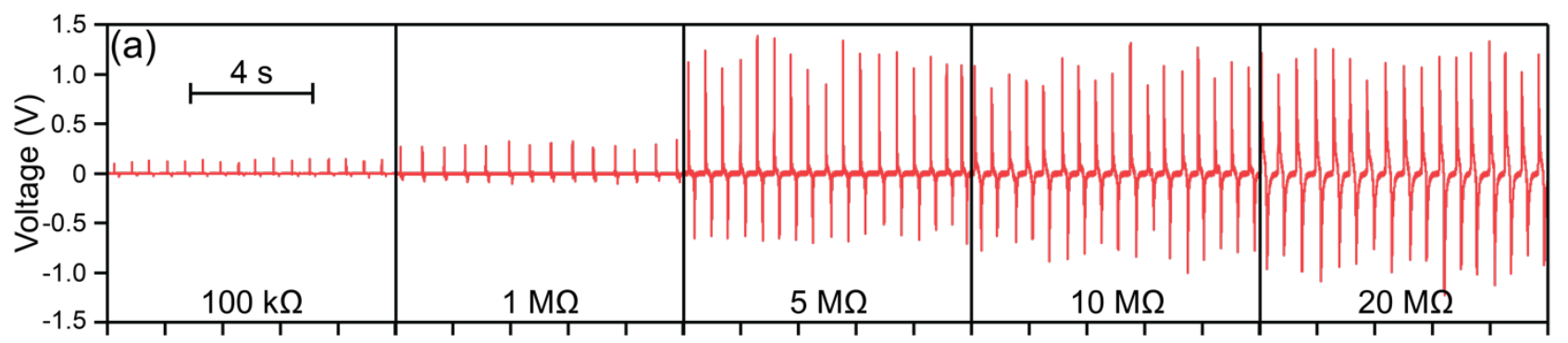

Time (s)
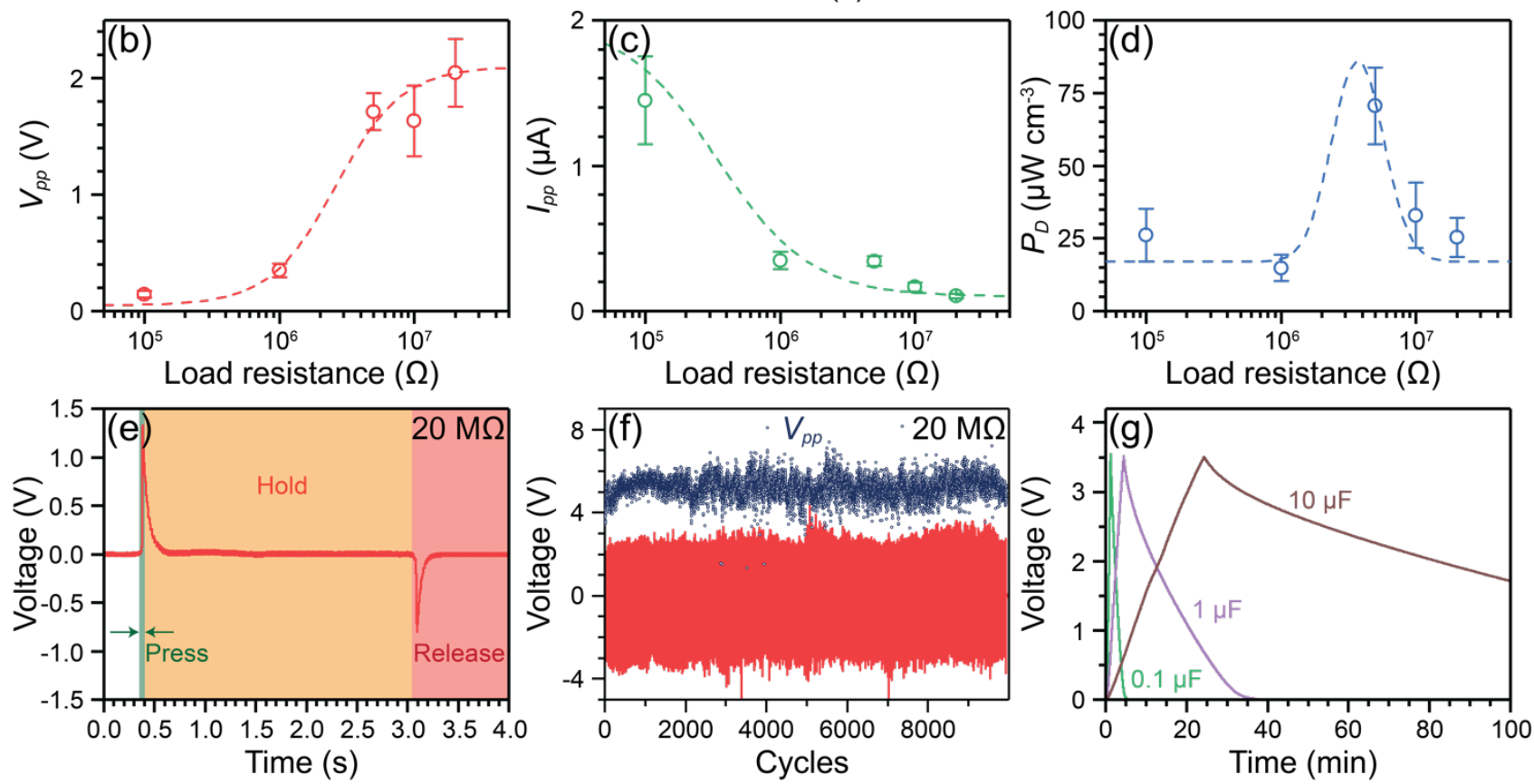

Fig. 8 Energy harvesting characteristics of a PEG fabricated from a recycled extrusion printed SWCNT/PVDF-TrFE (0.020 wt\%) film under low-force cyclic compression using finger pinching, showing (a) the generated voltage as a function of time with a load resistance between $100 \mathrm{k} \Omega$ and $20 \mathrm{M} \Omega$. (b) The peak to peak voltage $\left(V_{p p}\right)$ as a function of load resistance. (c) The peak to peak current $\left(I_{p p}\right)$ as a function of load resistance. (d) The volumetric power density $\left(P_{D}\right)$ as a function of load resistance. (e) The generated voltage at $20 \mathrm{M} \Omega$ as a function of time, where the PEG was compressed and held for approximately $1.7 \mathrm{~s}$ prior to release. (f) The generated voltage stability (red) and the $V_{p p}$ (blue) as a function of the number of compression cycles under a load resistance of $20 \mathrm{M \Omega}$.(g) The charging and discharging profiles of capacitors under $\mathrm{X} \mathrm{Hz}$ cyclic compression via a bridge rectifier.

\section{Conclusion}

Lowering the energy input required to manufacture polymerbased piezoelectric generators is crucial for their low-cost implementation into flexible and wearable electronics. This work has shown the low energy production of a transparent and flexible piezoelectric generator and is the first report of nonpoled fluoropolymer-based piezoelectric generators printed with a power density greater than $10 \mu \mathrm{W} \mathrm{cm}{ }^{-3}$. We have shown that by combining one-dimensional additives, in the form of SWCNTs, with polymer chain orientation via extrusion printing, we obtain figures of merit comparable to electrically poled PVDF-TrFE. Further, we suggest that this result arises from an increased dipole alignment of the crystalline phase relative to the SWCNT backbone, supported via molecular dynamics simulations. Further, we have shown the ability to recycle the SWCNT/PVDF-TrFE films using just a green solvent (acetone), giving a low energy tool to reshape the form factor of the piezoelectric generator. This form factor reshaping would allow piezoelectric generators to be made to size for wearable electronics, or for custom contours within the body to power devices such as pacemakers. The highly touted chemical stability of both SWCNTs and PVDF-TrFE means that this recycling process does not affect the fundamental chemistry, and hence the piezoelectric performance of the materials.

\section{Experimental}

\section{Ink preparation}

PVDF-TrFE powder ( $75 \mathrm{~mol} \%$ VDF and 25 mol\% TrFE, $M_{w}$ at 420 $\mathrm{kDa}, \rho$ at $1.49 \mathrm{~g} \mathrm{~cm}^{-3}$, Solvay, Italy), acetone (AR grade, ChemSupply Pty Ltd, Australia) and $N, N$-dimethylformamide (DMF) (ACS Reagent, Sigma-Aldrich, USA) were used without further purification. Single-walled carbon nanotubes (SWCNTs) with 1.2 $\mathrm{nm}$ average diameter and $3 \mu \mathrm{m}$ to $30 \mu \mathrm{m}$ length (99.9\% purity, Cheap Tubes Inc., USA) were dispersed via ultrasonication (Elmasonic S $30 \mathrm{H}$, Elma Schmidbauer, Germany) for $30 \mathrm{~min}$ in a solution consisting of acetone (60 vol\%) and DMF (40 vol\%) at $0.727 \mathrm{mg} \mathrm{mL}^{-1}$ to make a stock solution (corresponding to 0.200 
wt\% SWCNT/PVDF-TrFE solution). Further dilutions were then prepared from the stock solution at $0.363 \mathrm{mg} \mathrm{mL}^{-1}, 0.182 \mathrm{mg}$ $\mathrm{mL}^{-1}, 0.073 \mathrm{mg} \mathrm{mL}^{-1}, 0.036 \mathrm{mg} \mathrm{mL}^{-1}$ and $0.018 \mathrm{mg} \mathrm{mL}^{-1}$, corresponding to the relevant SWCNT concentration relative to PVDF-TrFE at $0.100 w t \%, 0.050 w t \%, 0.020 w t \%, 0.010 w t \%$ and 0.005 wt $\%$, respectively. The diluted solutions were further ultrasonicated for $5 \mathrm{~min}$. Subsequently, PVDF-TrFE powder (4 g) was added to each of the solutions $(9.333 \mathrm{~g})$ at $23{ }^{\circ} \mathrm{C}$ and magnetically stirred to dissolve the PVDF-TrFE. This made inks containing $30 \mathrm{wt} \%$ PVDF-TrFE relative to the solvent mixture with varying SWCNT concentrations ( 0.000 wt\% to 0.200 wt\%) relative to PVDF-TrFE.

\section{Printing and PEG manufacture}

The SWCNT/PVDF-TrFE (0.000 wt\% to 0.200 wt\%) inks were transferred into $30 \mathrm{~mL}$ dispensing barrels (Optimum, Nordson EFD, USA) and stored at $-5{ }^{\circ} \mathrm{C}$ prior to printing. The printing was carried out through a piston-driven 3D extrusion system (Bioplotter, EnvisionTEC, USA) through a nozzle with $0.20 \mathrm{~mm}$ internal diameter (SmoothFlow, Nordson EFD, USA) onto a clean glass substrate. Throughout printing, the barrel was kept at a temperature of $5^{\circ} \mathrm{C}$ and the stage at $23^{\circ} \mathrm{C}$. A one-layer film was printed following a raster pattern with $400 \mu \mathrm{m}$ spacing at $30 \mathrm{~mm} \mathrm{~s}^{-1} \mathrm{x}-\mathrm{y}$ speed under 1.7 Bar extrusion pressure with total dimensions of $30 \mathrm{~mm}$ in width and length. Subsequently, the films were placed under vacuum with an absolute pressure below $253 \mathrm{mbar}$ for $10 \mathrm{~min}$.

Post printing, the samples were stored in a sealed desiccator. For fabrication of the PEGs, electrodes were adhered to the extrusion printed films via sputter coating deposition (Q150T ES, Quorumtech, UK), consisting of a $2 \mathrm{~nm} \mathrm{Cr}$ layer and a $10 \mathrm{~nm}$ Au layer through a shadow mask with active area of $2.1 \mathrm{~cm}^{2}$ on both sides of the material. Wires, soldered to $\mathrm{Cu}$ foil tape with conductive adhesive (1181, 3M, USA), were adhered to both surfaces of the printed films, ensuring strong contact between the $\mathrm{Cu}$ tape and $\mathrm{Cr} / \mathrm{Au}$ coating. The films were finally encapsulated in insulating Kapton polyimide tape on both surfaces to complete the PEGs.

\section{Materials characterisation}

Viscosity of the inks was confirmed using a rheometer (MCR702, Anton Paar, Austria) with cone-plate geometry, with a cone diameter of $25 \mathrm{~mm}$ and angle at $2^{\circ}$. The measurements were obtained at a temperature of $5{ }^{\circ} \mathrm{C}$ in accordance with the printing barrel temperature by an upwards logarithmic ramp of shear rate from $0.01 \mathrm{~s}^{-1}$ to $200 \mathrm{~s}^{-1}$.

Optical properties, including transmittance and haze measurements of the printed PVDF-TrFE and SWCNT/PVDFTrFE films were performed on an ultraviolet, visible and near infrared (UV-vis-NIR) spectrophotometer (Lambda 950, Perkin Elmer, USA). The films were placed on the entry port of a $25 \mathrm{~cm}$ diameter integrating sphere accessory to capture the total light not absorbed by the material, as well as the proportion of light scattered at angles greater than $2^{\circ}$ from the beam direction.

The surface of the films was imaged using helium ion microscopy (HIM) (Orion NanoFab, Zeiss, Germany) to show representative distributions of the SWCNTS in the PVDF-TrFE. The micrographs were obtained with a $75 \mu \mathrm{m}$ field of view using a dwell time of $2 \mu \mathrm{s}$ and acceleration voltage of $25 \mathrm{kV}$.

Powder X-ray diffraction (XRD) patterns were obtained for the printed films using Bragg-Brentano geometry (D8 Advance, Bruker, Germany) using Cu-K $\alpha$ radiation ( $\lambda=1.54060 \AA$ ). The Bragg angle, $2 \theta$, was varied between $5^{\circ}$ and $70^{\circ}$ with a step size of $0.02^{\circ}$ and $1 \mathrm{~s}$ per step, with the sample rotated at $15 \mathrm{rpm}$. The films were placed on silicon low background holders for the measurements.

Raman spectroscopy (inVia, Renishaw, UK) was undertaken on the printed films, adhered to glass microscope slides using a $532 \mathrm{~nm}$ laser with a 1800 line $\mathrm{mm}^{-1}$ grating and a 50x objective. Each spectrum was centred at $1300 \mathrm{~cm}^{-1}$ and obtained using a laser power of $1.68 \mathrm{~mW}$ with an exposure time of $0.15 \mathrm{~s}$ accumulated over 1000 scans. Confocal images were obtained $0.5 \mathrm{~s}$ before scanning and $0.5 \mathrm{~s}$ after the scan was finished in order to ensure the sample remained stable under laser irradiation.

Differential scanning calorimetry (DSC) traces were obtained using a simultaneous thermal analyser (STA 8000, Perkin Elmer, USA). Samples weighing between $10 \mathrm{mg}$ and 15 $\mathrm{mg}$ were placed into a ceramic pan and heated to $180^{\circ} \mathrm{C}$ at a ramp rate of $10^{\circ} \mathrm{C} \mathrm{min}-1$. The furnace was continuously purged with nitrogen of ultra-high purity at a rate of $20 \mathrm{~mL} \mathrm{~min}^{-1}$, and equilibrated for 30 min between scans.

Fourier transform infrared (FTIR) analysis (ALPHA II, Bruker, USA) was performed on the printed films in attenuated total reflectance (ATR) mode. The absorbance was taken as an average of 128 individual scans with a resolution of $2 \mathrm{~cm}^{-1}$ between $600 \mathrm{~cm}^{-1}$ and $4000 \mathrm{~cm}^{-1}$.

The thickness, and consequently the volume of the printed films was determined from the final dry mass using the density of PVDF-TrFE as $1.49 \mathrm{~g} \mathrm{~cm}^{-3}$. The mass was determined as an average of a minimum of three printed films for each SWCNT concentration and the length and width were taken as $3 \mathrm{~cm}$ to obtain the thickness.

\section{Piezoresponse force microscopy characterisation}

Piezoresponse force microscopy (PFM) was undertaken (Cypher $\mathrm{ES}$, Asylum Research, USA) at $23^{\circ} \mathrm{C}$ on the printed films for the determination of nanoscale polarisation characteristics. Cantilevers (Econo-SCM-PIC, Asylum Research, USA) with $\mathrm{Cr} / \mathrm{Pt}$ conductive coating, tip radius below $25 \mathrm{~nm}$ and spring constant of $0.2 \mathrm{~N} \mathrm{~m}^{-1}$ were used for the PFM measurements. The scans were completed on an area of $5 \mu \mathrm{m} \times 5 \mu \mathrm{m}$ at 256 pixels per line, corresponding to approximately $20 \mathrm{~nm}$ per pixel.

The PFM was carried out in contact lithography mode, whereby a potential was applied to the sample in pre-defined patterns and the amplitude and phase changes relative to the unbiased areas were observed. The voltage was swept in increments of approximately $2 \mathrm{~V}$ from $20 \mathrm{~V}$ to $-20 \mathrm{~V}$ on each scan line, with a scan rate of $0.2 \mathrm{~Hz}$. Each voltage step was applied to an area of $195 \mathrm{~nm}$ in the $x$ direction and $3.906 \mu \mathrm{m}$ in the $y$ direction. 
The resultant amplitude and phase data were then correlated to the corresponding voltage for each pixel on the scan. Dual AC resonance tracking (DART-PFM) was then undertaken at $1 \mathrm{~Hz}$ scan rate to ensure the stability of the material.

\section{Energy harvesting analysis}

Energy harvesting characterisation was completed to determine the macroscale polarisation and ultimate energy harvesting potential of the PVDF-TrFE and SWCNT/PVDF-TrFE PEGs. The voltage output of the PEGs was measured using an oscilloscope with $10 \mathrm{M} \Omega$ input impedance (TDS2002B, Tektronix, USA) under cyclic compression (5963, Instron, USA) with a constant frequency of approximately $5 \mathrm{~Hz}$ and controlled strain rate. The load cell was rated at $1 \mathrm{kN}$. The speed was set at $10 \mathrm{~mm} \mathrm{~s}^{-1}$, with a $50 \mathrm{~N}$ maximum set force. Rubber mats were placed between the PEG and the instrument for the measurement, with a thickness of $\sim 1 \mathrm{~mm}$ each.

Further characterisation of the power and power density was carried out in a similar manner, while resistive loads were attached in parallel and the voltage output was measured under cyclic compression.

\section{Molecular dynamics modelling}

All-atom Molecular Dynamics (MD) simulations were employed to investigate the preferred conformation of the PVDF-TrFE chains on various SWCNTs. SWCNTs with two chiralities were simulated, a $(8,8)$ and a $(12,0)$ CNT corresponding to the armchair and the zig-zag configuration, respectively. The SWCNT systems had a length of $10 \mathrm{~nm}$ each and diameters of $1.084 \mathrm{~nm}$ and 0.993 for the $(8,8)$ and $(12,0)$ chiralities, respectively. For each SWCNT, two PVDF-TrFE chains containing $21 \mathrm{VDF}$ and 9 TrFE monomers were introduced in the simulation cell, corresponding to molar concentrations of $70 \mathrm{~mol} \% \mathrm{VDF}$ and 30 mol\% TrFE.

The SWCNT and co-polymer systems were simulated in the NVT (constant number of particles, volume, and temperature) ensemble at $300 \mathrm{~K}$ for at least $4 \mathrm{~ns}$ under vacuum. For statistically relevant results three simulations of the same system were carried out at the same conditions with different initial configurations, meaning that new positions and velocities were assigned to each atom. The equations of motion were integrated using the velocity-Verlet algorithm ${ }^{79}$ with a time step of $1 \mathrm{fs}$ using the polymer consistent force field (PCFF) for both the CNT and the co-polymers. Partial charges were assigned to each atom of the CNT and the PVDF-TrFE using the bond increments method. The PCFF interatomic potential was validated with the density of bulk PVDF-TrFE melts at $230{ }^{\circ} \mathrm{C}$. Fig. S15 shows the monotonic increase of the PVDF-TrFE density $\rho$ with the molecular weight $M_{w}$, attaining a plateau at approximately $1.57 \mathrm{~g} \mathrm{~cm}^{-3}$ for PVDF-TrFE melts with $M_{w}$ greater than $10,000 \mathrm{~g} \mathrm{~mol}^{-1}$. The MD-obtained density was found to be in accordance (deviation below $5.5 \%$ ) with the experimental value $\left(\rho_{\text {bulk }}=1.49 \mathrm{gr} \mathrm{cm}^{-3}\right)$ of the bulk PVDF-TrFE copolymer with the same composition (70 mol\% VDF and $30 \mathrm{~mol} \% \mathrm{TrFE}$ ), validating the interatomic potential used in the present simulations. The MD simulations were performed on the University of Melbourne's High-Performance Computing system using the LAMMPS MD code. ${ }^{80}$

\section{Recyclability testing}

Four printed SWCNT/PVDF-TrFE (0.020 wt\%) films, with a total mass of $0.214 \mathrm{~g}$ were placed into a dispensing barrel. Acetone, with a total volume of $0.905 \mathrm{~mL}$ was added to the barrel, corresponding to $23 \mathrm{wt} \%$ polymer loading. The barrel was sealed, gently shaken and subsequently held at $-5^{\circ} \mathrm{C}$ for $12 \mathrm{~h}$, to ensure a homogeneous mixture of the ink. Printing of the resulting ink was undertaken in similar conditions to the initial printing cycle with the exception of the extrusion pressure, which was decreased to 0.3 Bar.

The energy harvesting experiment for the recycled and reprinted SWCNT/PVDF-TrFE (0.020 wt\%) PEG used low-force cyclic finger pinching as input force. The load resistance was set using a resistance decade (M642, Meatest, Czech Republic) and the output voltage measured using a potentiostat (VMP3, BioLogic, France) in open circuit voltage mode with an input impedance of $10^{14} \Omega$.

\section{Conflicts of interest}

There are no conflicts to declare.

\section{Acknowledgements}

This research was supported by the Australian Government through the Australian Research Council's Linkage Projects funding scheme (LP160100071) and Future Fellowships funding scheme (FT130100211). This work was performed in part at the Materials Characterisation and Fabrication Platform (MCFP) at the University of Melbourne and the Victorian Node of the Australian National Fabrication Facility (ANFF).

\section{References}

1 Y. Wurmser, Wearables 2019: Advanced Wearabled Pick Up Pace as Fitness Trackers Slow, 2019.

2 Y. Huang, L. Zhao, L. Li, M. Xie, F. Wu and R. Chen, Adv. Mater., 2019, 31, 1808393.

3 Y. Yang and W. Gao, Chem. Soc. Rev., 2019, 48, 1465-1491.

4 H. Wang, A. Guerrero, A. Bou, A. M. Al-Mayouf and J. Bisquert, Energy Environ. Sci., 2019, 12, 2054-2079.

5 Y. Wang, L. Yang, X.-L. Shi, X. Shi, L. Chen, M. S. Dargusch, J. Zou and Z.-G. Chen, Adv. Mater., 2019, 31, 1807916.

6 C. R. Bowen, J. Taylor, E. Leboulbar, D. Zabek, A. Chauhan and R. Vaish, Energy Environ. Sci., 2014, 7, 3836-3856.

7 N. A. Shepelin, A. M. Glushenkov, V. C. Lussini, P. J. Fox, G. W. Dicinoski, J. G. Shapter and A. V Ellis, Energy Environ. Sci., 2019, 12, 1143-1176.

8 C. Kolm and P. H. Fowler, US Pat., 3239678A, 1966.

9 K. S. Ramadan, D. Sameoto and S. Evoy, Smart Mater. Struct., 2014, 23, 033001.

10 F. R. Fan, W. Tang and Z. L. Wang, Adv. Mater., 2016, 28, 4283-4305.

11 N. Weber, Y.-S. Lee, S. Shanmugasundaram, M. Jaffe and T. L. Arinzeh, Acta Biomater., 2010, 6, 3550-3556. 
12 J. Li, L. Kang, Y. Yu, Y. Long, J. J. Jeffery, W. Cai and X. Wang, Nano Energy, 2018, 51, 728-735.

13 V. Sencadas, S. Lanceros-Méndez and J. F. Mano, Thermochim. Acta, 2004, 424, 201-207.

14 D. B. Deutz, J.-A. Pascoe, B. Schelen, S. Van Der Zwaag, D. M. De Leeuw and P. Groen, Mater. Horizons, 2018, 5, 444-453.

15 P. Martins, A. C. Lopes and S. Lanceros-Mendez, Prog. Polym. Sci., 2014, 39, 683-706.

16 J. C. Dias, A. C. Lopes, B. Magalhães, G. Botelho, M. M. Silva, J. M. S. S. Esperança and S. Lanceros-Mendez, Polym. Test., 2015, 48, 199-205.

17 J. Y. Lim, S. Kim and Y. Seo, AIP Conf. Proc., 2015, 1664, 070006.

18 H. M. Ning, N. Hu, T. Kamata, J. H. Qiu, X. Han, L. M. Zhou, C. Chang, Y. Liu, L. K. Wu, J. H. Qiu, H. L. Ji, W. X. Wang, Y. Zemba, S. Atobe, Y. Li, Alamusi and H. Fukunaga, Smart Mater. Struct., 2013, 22, 065011.

19 R. Su, J.-K. Tseng, M.-S. Lu, M. Lin, Q. Fu and L. Zhu, Polymer., 2012, 53, 728-739.

20 Measurement Specialties Inc., Piezo Film Sensors Technical Manual, Norristown, PA, 1999.

21 M. S. Sebastian, A. Larrea, R. Gonçalves, T. Alejo, J. L. Vilas, V. Sebastian, P. Martins and S. Lanceros-Mendez, RSC Adv., 2016, 6, 113007-113015.

22 N. Jia, Q. Xing, X. Liu, J. Sun, G. Xia, W. Huang and R. Song, J. Colloid Interface Sci., 2015, 453, 169-176.

23 C. Ben Osman, S. Nowak, A. Garcia-Sanchez, Y. Charles, S. Ammar, S. Mercone and F. Mammeri, Eur. Polym. J., 2016, 84, 602-611.

24 M. M. Alam, A. Sultana and D. Mandal, ACS Appl. Energy Mater., 2018, 1, 3103-3112.

25 X. Hu, X. Yan, L. Gong, F. Wang, Y. Xu, L. Feng, D. Zhang and Y. Jiang, ACS Appl. Mater. Interfaces, 2019, 11, 7379-7386.

26 S. Manna and A. K. Nandi, J. Phys. Chem. C, 2007, 111, 1467014680.

27 G. Mago, F. T. Fisher and D. M. Kalyon, J. Nanosci. Nanotechnol., 2009, 9, 3330-3340.

28 S. Chen, X. Tao, W. Zeng, B. Yang and S. Shang, Adv. Energy Mater., 2017, 7, 1601569.

29 L. He, J. Sun, X. Wang, L. Yao, J. Li, R. Song, Y. Hao, Y. He and W. Huang, J. Colloid Interface Sci., 2011, 363, 122-128.

30 Z. H. Liu, C. T. Pan, L. W. Lin and H. W. Lai, Sensors Actuators, A Phys., 2013, 193, 13-24.

31 A. Mandal and A. K. Nandi, ACS Appl. Mater. Interfaces, 2013, 5, 747-760.

32 L. He, G. Xia, J. Sun, Q. Zhao, R. Song and Z. Ma, J. Colloid Interface Sci., 2013, 393, 97-103.

33 X. Guan, Y. Zhang, H. Li and J. Ou, Sensors Actuators, A Phys., 2013, 194, 228-231.

34 W. Huang, Z. Li, X. Chen, P. Tian, J. Lu, Z. Zhou, R. Huang, D. Hui, L. He, C. Zhang and X. Wang, Compos. Part B Eng., 2014, 62, 126-136.

35 A. Sarvi, V. Chimello, A. B. Silva, R. E. S. Bretas and U. Sundararaj, Polym. Compos., 2014, 35, 1198-1203.

36 F. Bai, G. Chen, M. Nie and Q. Wang, RSC Adv., 2015, 5, 5417154174.

37 R. M. Habibur, U. Yaqoob, S. Muhammad, A. S. M. I. Uddin and H. C. Kim, Mater. Chem. Phys., 2018, 215, 46-55.

38 R. Tian, Q. Xu, C. Lu, X. Duan and R.-G. Xiong, Chem. Commun., 2017, 53, 7933-7936.

39 S. Lee and Y. Lim, Macromol. Mater. Eng., 2018, 303, 1700588.

40 P. C. Sherrell, A. Cieślar-Pobuda, M. S. Ejneby, L. Sammalisto, A. Gelmi, E. de Muinck, J. Brask, M. J. Łos and M. Rafat, Macromol. Biosci., 2017, 17, 1600446.

41 V. Flexer, J. Chen, B. C. Donose, P. Sherrell, G. G. Wallace and J. Keller, Energy Environ. Sci., 2013, 6, 1291-1298.

42 P. M. Ajayan, L. S. Schadler, C. Giannaris and A. Rubio, Adv. Mater., 2000, 12, 750-753.
43 L. Wu, W. Yuan, T. Nakamura, S. Atobe, N. Hu, H. Fukunaga, C. Chang, Y. Zemba, Y. Li, T. Watanabe, Y. Liu, Alamusi, H. Ning, J. Li, H. Cui and Y. Zhang, Adv. Compos. Mater., 2013, 22, 4963.

44 J. Kim, K. J. Loh and J. P. Lynch, in Sensors and Smart Structures for Civil, Mechanical, and Aerospace Systems 2008, Proc. SPIE 6932, San Diego, California, United States, 2008, p. 693232.

45 S. Bodkhe, G. Turcot, F. P. Gosselin and D. Therriault, ACS Appl. Mater. Interfaces, 2017, 9, 20833-20842.

46 S. Bodkhe, P. S. M. Rajesh, F. P. Gosselin and D. Therriault, ACS Appl. Energy Mater., 2018, 1, 2474-2482.

47 N. A. Shepelin, V. C. Lussini, P. J. Fox, G. W. Dicinoski, A. M. Glushenkov, J. G. Shapter and A. V Ellis, MRS Commun., 2019, 9, 159-164.

48 Z. Zhu, S.-Z. Guo, T. Hirdler, C. Eide, X. Fan, J. Tolar and M.C. McAlpine, Adv. Mater., 2018, 30, 1707495.

49 M.-C. García-Gutiérrez, A. Linares, I. Martín-Fabiani, J. J. Hernández, M. Soccio, D. R. Rueda, T. A. Ezquerra and M. Reynolds, Nanoscale, 2013, 5, 6006-6012.

50 E. Bellet-Amalric and J. F. Legrand, Eur. Phys. J. B, 1998, 3, 225-236.

51 C. J. L. Constantino, A. E. Job, R. D. Simões, J. A. Giacometti, V. Zucolotto, O. N. Oliveira Jr., G. Gozzi and D. L. Chinaglia, Appl. Spectrosc., 2005, 59, 275-279.

52 S. Zhang, H. Wang, D. Xu, W. Yang, P. Tang and Y. Bin, J. Appl. Polym. Sci., 2016, 133, 43605.

53 M. T. Riosbaas, K. J. Loh, G. O'Bryan and B. R. Loyola, in Sensors and Smart Structures Technologies for Civil, Mechanical, and Aerospace Systems 2014, Proc. SPIE 9061 San Diego, California, United States, 2014, p. $90610 Z$.

54 A. Ferrari and J. Robertson, Phys. Rev. B - Condens. Matter Mater. Phys., 2000, 61, 14095-14107.

55 X. Chen, J. Shao, N. An, X. Li, H. Tian, C. Xu and Y. Ding, J. Mater. Chem. C, 2015, 3, 11806-11814.

56 P. Cebe and J. Runt, Polymer., 2004, 45, 1923-1932.

57 X. Cai, T. Lei, D. Sun and L. Lin, RSC Adv., 2017, 7, 1538215389.

58 S. K. Ghosh and D. Mandal, Nano Energy, 2018, 53, 245-257.

59 J. Gregorio R. and M. Cestari, J. Polym. Sci. Part B Polym. Phys., 1994, 32, 859-870.

60 F. Xu, K. Zhang, Y. Zhou, Z. Qu, H. Wang, Y. Zhang, H. Zhou and C. Yan, RSC Adv., 2017, 7, 17038-17043.

61 X. Li, Y.-F. Lim, K. Yao, F. E. H. Tay and K. H. Seah, Phys. Chem. Chem. Phys., 2013, 15, 515-520.

62 S. Florczak, T. Lorson, T. Zheng, M. Mrlik, D. W. Hutmacher, M. J. Higgins, R. Luxenhofer and P. D. Dalton, Polym. Int., 2019, 68, 735-745.

63 U. Yaqoob, R. M. Habibur, M. Sheeraz and H. C. Kim, Compos. Part B Eng., 2019, 159, 259-268.

64 M. Pusty, L. Sinha and P. M. Shirage, New J. Chem., 2019, 43 , 284-294.

65 P. Thakur, A. Kool, N. A. Hoque, B. Bagchi, F. Khatun, P. Biswas, D. Brahma, S. Roy, S. Banerjee and S. Das, Nano Energy, 2018, 44, 456-467.

66 A. Biswas, S. Garain and D. Mandal, AIP Conf. Proc., 2017, 1832, 040024.

67 M. Pusty, A. Sharma, L. Sinha, A. Chaudhary and P. Shirage, ChemistrySelect, 2017, 2, 2774-2782.

68 P. Adhikary, S. Garain, S. Ram and D. Mandal, J. Polym. Sci. Part B Polym. Phys., 2016, 54, 2335-2345.

69 S. K. Karan, R. Bera, S. Paria, A. K. Das, S. Maiti, A. Maitra and B. B. Khatua, Adv. Energy Mater., 2016, 6, 1601016.

70 R. A. Whiter, V. Narayan and S. Kar-Narayan, Adv. Energy Mater., 2014, 4, 1400519.

71 B. Stadlober, M. Zirkl and M. Irimia-Vladu, Chem. Soc. Rev., 2019, 48, 1787-1825. 
72 A. Tamang, S. K. Ghosh, S. Garain, M. M. Alam, J. Haeberle, K. Henkel, D. Schmeisser and D. Mandal, ACS Appl. Mater. Interfaces, 2015, 7, 16143-16147.

73 P. G. Jessop, D. A. Jessop, D. Fu and L. Phan, Green Chem., 2012, 14, 1245-1259.

74 K. Alfonsi, J. Colberg, P. J. Dunn, T. Fevig, S. Jennings, T. A. Johnson, H. P. Kleine, C. Knight, M. A. Nagy, D. A. Perry and M. Stefaniak, Green Chem., 2008, 10, 31-36.

75 L. Zhang, J. Gui, Z. Wu, R. Li, Y. Wang, Z. Gong, X. Zhao, C. Sun and S. Guo, Nano Energy, 2019, 65, 103924.

76 K.-I. Park, J. H. Son, G.-T. Hwang, C. K. Jeong, J. Ryu, M. Koo, I. Choi, S. H. Lee, M. Byun, Z. L. Wang and K. J. Lee, Adv. Mater., 2014, 26, 2514-2520.

77 Y. Zhang, M. Wu, Q. Zhu, F. Wang, H. Su, G. Li, C. Diao, H. Zheng, Y. Wu and Z.L. Wang, Adv. Funct. Mater., 2019, 29, 1904259.

78 V. Sencadas, C. Garvey, S. Mudie, J.J.K. Kirkensgaard, G. Gouadec and S. Hauser, Nano Energy, 2019, 66, 104106

79 W. C. Swope, H. C. Andersen, P. H. Berens and K. R. Wilson, J. Chem. Phys., 1982, 76, 637-649.

80 S. Plimpton, J. Comput. Phys., 1995, 117, 1-19. 


\section{University Library}

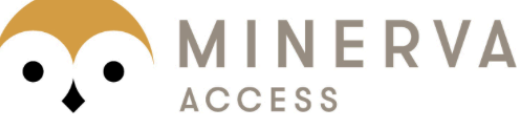

A gateway to Melbourne's research publications

Minerva Access is the Institutional Repository of The University of Melbourne

Author/s:

Shepelin, NA;Sherrell, PC;Goudeli, E;Skountzos, EN;Lussini, VC;Dicinoski, GW;Shapter, JG;Ellis, AV

Title:

Printed recyclable and self-poled polymer piezoelectric generators through single-walled carbon nanotube templating

Date:

2020-03-01

Citation:

Shepelin, N. A., Sherrell, P. C., Goudeli, E., Skountzos, E. N., Lussini, V. C., Dicinoski, G. W., Shapter, J. G. \& Ellis, A. V. (2020). Printed recyclable and self-poled polymer piezoelectric generators through single-walled carbon nanotube templating. Energy and Environmental Science, 13 (3), pp.868-883. https://doi.org/10.1039/C9EE03059J.

Persistent Link:

http://hdl.handle.net/11343/241899 